Rechtsfragen des Internet-Vertriebs

von Versicherungsdienstleistungen

Carsten Hoppmann / Flemming Moos

Arbeitspapier Nr. 72 


\section{Rechtsfragen des Internet-Vertriebs von Versicherungsdienstleistungen}

von Carsten Hoppmann ${ }^{*}$ und Flemming Moos ${ }^{* *}$, Osnabrück

Rechtsfragen der kommerziellen Internet-Nutzung

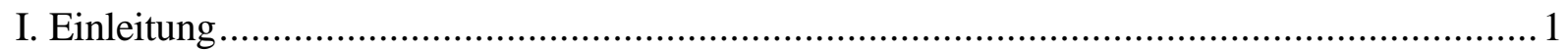

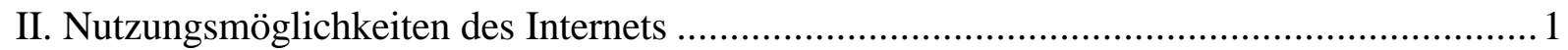

III. Wirtschaftliche Chancen des Internets für Versicherungsunternehmen ............................ 4

IV. Allgemeine Rechtsprobleme bei der Internet-Nutzung ............................................... 7

1. Rechtliche Einordnung einer Unternehmens-Homepage .............................................. 8

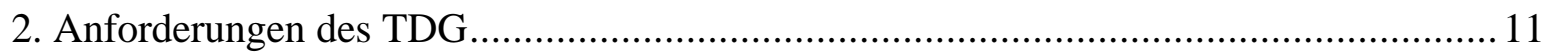

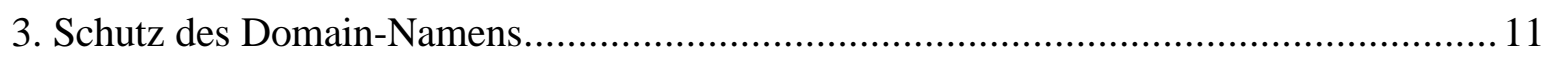

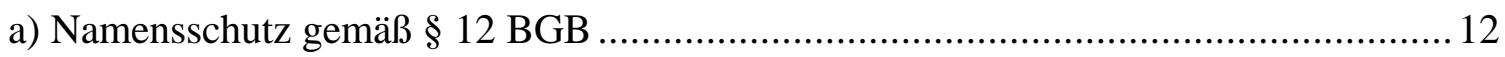

b) Schutz als geschäftliche Bezeichnung gemäß § 5 MarkenG .................................... 12

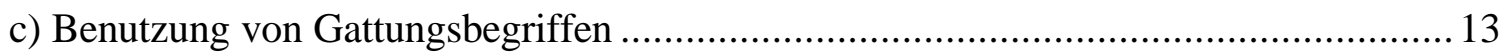

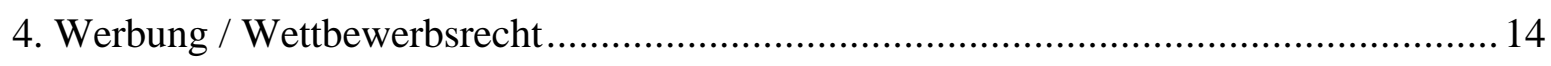

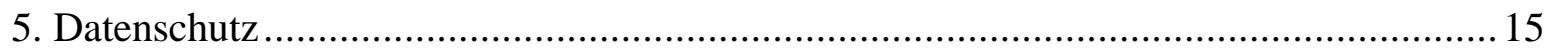

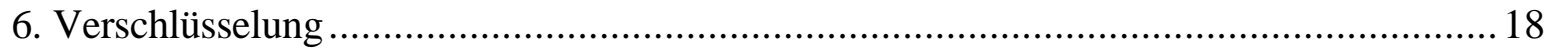

V. Abschluß von Versicherungsverträgen im Internet..................................................... 19

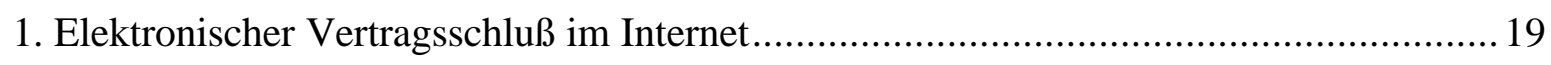

a) Die Homepage als Angebot oder als invitatio ad offerendum ................................2 20

b) Angebot und Annahme unter Abwesenden oder Anwesenden ..................................20

c) Einbeziehung von Allgemeinen Versicherungsbedingungen als AGB .......................22

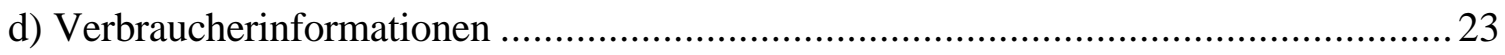

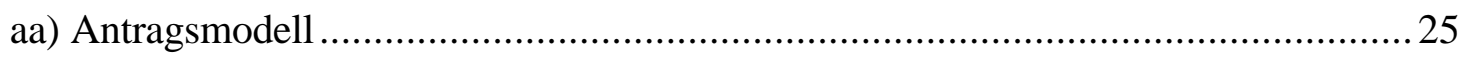

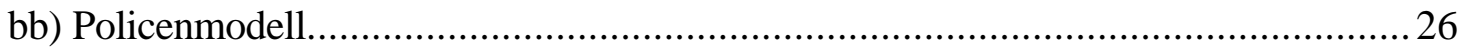

e) Das Widerrufs- und Rücktrittsrecht gemäß $\S 8$ Abs. 4 und 5 VVG ............................2 28

Doktorand am Institut für Handels- und Wirtschaftsrecht der Universität Osnabrück.

** Doktorand am Lehrstuhl für Öffentliches Recht, Europarecht und Völkerrecht der Universität Osnabrück. 


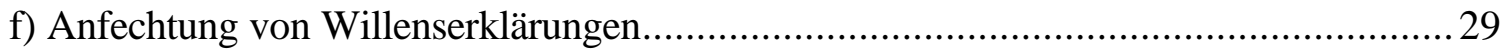

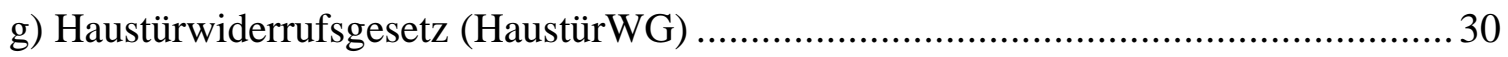

2. Sicherung von Identität des Vertragspartners und Authentizität des Vertragsinhalts .......30

3. Gerichtliche Durchsetzbarkeit / Schriftformerfordernis ............................................... 31

VI. Anwendbarkeit der Fernabsatzrichtlinie auf Versicherungsverträge .............................. 33

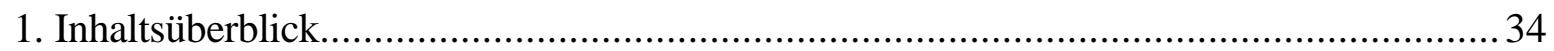

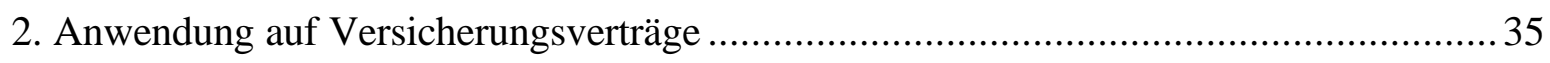

3. Geplante Fernvertragsrichtlinie über Finanzdienstleistungen ................................... 36

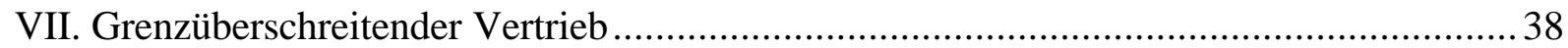

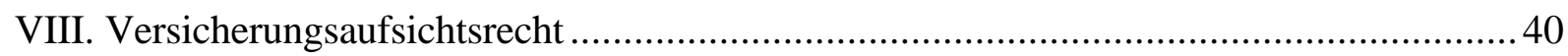

1. Vertrieb von Versicherungsdienstleistungen im Inland ............................................ 40

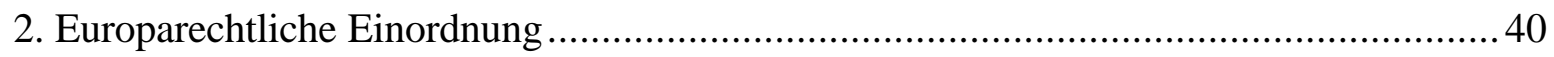

3. Rechtliche Einordnung von Unternehmen aus Drittstaaten als

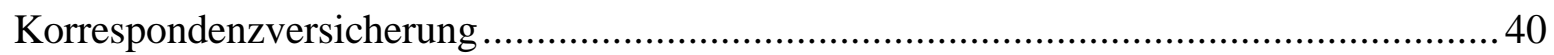

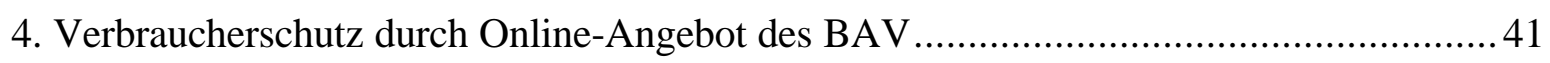

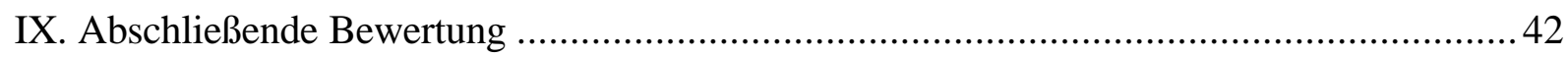




\section{Einleitung}

Während in vielen Wirtschaftsbereichen der Online-Vertragsabschluß trotz der bestehenden rechtlichen und technischen Unwägbarkeiten eine Alltäglichkeit geworden ist, liegen die Dinge in der Versicherungswirtschaft anders. Es sind zwar heute fast alle großen Versicherungsunternehmen im Netz; die überwiegende Zahl der Angebote bleibt aber noch weit hinter dem technisch bereits Möglichen zurück ${ }^{1}$. Als Grund für diese Zurückhaltung führen die Versicherungsunternehmen neben Sicherheitsrisiken vor allem rechtliche Probleme mit dem neuen Medium ins Feld ${ }^{2}$. Dieser Beitrag macht es sich deshalb zur Aufgabe, zunächst die Nutzungsmöglichkeiten des Internets für Versicherungsunternehmen darzustellen und die wirtschaftlichen Chancen des Internets für Versicherungsunternehmen aufzuzeigen, um dann eingehend die rechtlichen Probleme bei der Nutzung dieses neuen Mediums zum Abschluß und zur Abwicklung von Versicherungsverträgen zu erörtern. Auf diese Weise soll eine juristische Entscheidungshilfe für den Einsatz des Internets als Vertriebsmedium in der Versicherungswirtschaft gegeben werden.

\section{Nutzungsmöglichkeiten des Internets}

In den vergangenen Jahren hat sich infolge der Deregulierung des Versicherungsrechts ${ }^{3}$, der

1 In einer Reihe von Studien wurden bereits die Nutzungsmöglichkeiten des Internets für Versicherungsunternehmen erörtert: Theil, JFB 1996, S. 155 ( 156 ff); Schöffski/Samusch, ZVersWiss 1997, S. 171 (190); Blawath VW 1997, S. 518 ff.; Studie der PG\&P:Consulting, Versicherungen im Internet, Leinfelden 1998; Studie von Andersen Consulting, Paradigmenwechsel in der Versicherungswirtschaft, Ergebnisse abgedruckt in: Versicherungskaufmann, 3/1998, S. 22.

2 Vgl. Blawath, VW 1997, S. 518 (522). Aus den Sicherheitsrisiken und den rechtlichen Problemen im Internet können sich Versicherungsfälle ergeben, die in den meisten bestehenden Policen der Unternehmen nicht erfaßt sind, vgl. Knospe, ZfV 1998, S. 372 f.

3 Hier ist vor allem die Realisierung des europäischen Binnenmarktes für Finanzdienstleistungen, der auf den Grundsätzen der Herkunftslandkontrolle und der gegenseitigen Anerkennung beruht, zu nennen, vgl. Grünbuch der Kommission - Finanzdienstleistungen: Wahrung der Verbraucherinteressen, KOM (96) 209 endg. v. 22.5.1996, S. 3; grundlegend: Stadler, Die Liberalisierung des Dienstleistungshandels am Beispiel der Versicherungen, Berlin 1992. 
zunehmenden Globalisierung ${ }^{4}$ und dem damit verbundenen verstärkten Auftreten ausländischer Versicherungen auf dem heimischen Markt die Wettbewerbslandschaft grundlegend verändert. Versicherungsunternehmen und Verbraucher mußten sich auf die neue Situation einstellen. Eine Vielzahl neuer Produkte ist entwickelt worden, und die individuellen Bedürfnisse der Verbraucher können besser berücksichtigt werden. Die Tarifunterschiede zwischen Versicherungen sind durch den Wegfall der Bedingungs- und Tarifgenehmigung, die eine vereinheitlichende Wirkung hatten, deutlich geworden. Für die Versicherungsunternehmen ist ein starker Kostendruck entstanden. Die neuen Versicherungsprodukte, die größeren Tarifunterschiede und eine Vielzahl von Rabatten haben dazu geführt, daß es für den Verbraucher nahezu unmöglich ist, den Versicherungsmarkt zu überblicken.

Das Internet könnte zur Lösung der bestehenden Probleme beitragen. Es ist ein überaus kostengünstiges Marketing- und Vertriebsmedium, und der Verbraucher könnte sich in einer sehr kurzen Zeit einen umfassenden Überblick über den Versicherungsmarkt verschaffen. Der zeitaufwendige Weg zu einem Agenten entfällt für ihn.

Einer Umfrage des Gesamtverbandes der Deutschen Versicherungswirtschaft (GDV) zufolge sind über $50 \%$ aller Versicherungsunternehmen gegenwärtig im Internet vertreten. Von den bedeutenden Versicherungsunternehmen sind fast alle in diesem neuen Medium präsent ${ }^{5}$. Die Funktionen und Nutzen der Online-Angebote unterscheiden sich aber sehr stark zwischen den einzelnen Unternehmen.

Die meisten Unternehmen nutzen das Internet $\mathrm{zu}$ Zwecken der Werbung und der Öffentlichkeitsarbeit. Das Versicherungsunternehmen wird mit seiner Geschichte,

$4 \quad$ Als die wesentliche Triebfeder dürften hier die Verhandlungen im Rahmen der WTO anzusehen sein.

Ende 1997 ist von über 100 WTO-Mitgliedern ein Abkommen zur Liberalisierung der Finanzdienstleistungen unterzeichnet worden, daß als fünftes Protokoll zum GATS am 1.3.1999 in Kraft treten soll; s. dazu: Hilpold, RIW 1998, S. 90 (98 f.); Sorsa, The GATS Agreement on Financial Services, IMF Working Paper WP/97/55, Genf 1997; Zutshi, Aspects of the Final Outcome of the Negotiations on Financial Services of the Uruguay Round, UNCTAD Discussion Papers No. 109, Genf 1995.

5 Vgl. die Übersicht mit Bewertung der Web-Angebote bei Kahlen, Capital 12/1997 und die nahezu vollständige Linksammlung $\mathrm{zu}$ deutschen Versicherungsunternehmen auf einer Site der Fachhochschule Köln: http://www.fh-Koeln.de/fb-vw/links/links.htm. 
Unternehmensphilosophie, Mitarbeitern und den Produkten vorgestellt. Neben der schlichten Präsenz im Internet und der damit verbundenen Nutzung des Internets als Marketing-Medium bieten die meisten Internet-Angebote dem Kunden die Möglichkeit, per E-Mail mit der Versicherung in Kontakt zu treten. Auf diesem Weg kann er dann Vertragsunterlagen, Angebote und weitere Werbung anfordern. Ferner kann der Kunde mit Hilfe des world wide web (www.) auch herausfinden, welcher Versicherungsvertreter ${ }^{6}$ in seiner Nähe für ihn zuständig ist. Die hier genannten Nutzungsmöglichkeiten gehören zum Standard des InternetAuftritts von Versicherungsunternehmen.

Die weiteren Nutzungsmöglichkeiten des Internets werden dagegen nur von verhältnismäßig wenigen Versicherungsunternehmen in Deutschland in Anspruch genommen. Hierzu gehört die Berechnung einer individuellen Prämie, die Online-Schadensmeldung oder auch eine OnlineGesundheitsprüfung im Rahmen einer Lebensversicherung. Daneben haben einige Versicherungen besondere Produkte für das Internet entwickelt. Dazu gehören z.B. standardisierte Risikolebensversicherungen, die ausschließlich und $\mathrm{zu}$ besonders günstigen Tarifen im Internet angeboten werden. Allgemein wird den Assekuranzdienstleistungen eine hohe Eignung für den Vertrieb mittels Internet zugeschrieben ${ }^{7}$. Vergleichende Studien belegen diese Einschätzung anhand der strategischen Planung von Versicherungsunternehmen. Danach schreiben die Versicherungsunternehmen dem www. für die Distributionspolitik eine von Jahr zu Jahr wachsende Bedeutung $\mathrm{zu}^{8}$. Die Abwicklung erfolgt aber im Regelfall (noch) nicht vollständig über das Internet. Der Kunde fordert lediglich per E-Mail die Vertragsunterlagen an. Der eigentliche Vertragsabschluß findet dann außerhalb des virtuellen Marktes statt. Der Kunde muß bei all diesen Nutzungsmöglichkeiten persönliche Daten, die als höchst vertraulich einzustufen sind (z. B. Gesundheitsdaten), über das Medium Internet an das Versicherungsunternehmen übermitteln. Die Gewährleistung eines ausreichenden Datenschutzes ist deshalb ein zentrales Anliegen.

6 Zur Anbindung der Außendienstmitarbeiter durch Internet-Kommunikation s. Schmidt, Die Datenverarbeitung im Versicherungsbetrieb, Wiesbaden 1993, S. 42 ff.

$7 \quad$ Fritz, VW 1998, S. 1431 (1431); Schinzer, Auswahl einer geeigneten Electronic Commerce-Strategie, in: Thome/Schinzer, 1997, S. 19 (24).

$8 \quad$ Vgl. Fritz, VW 1998, S. 1431 (1432). 
Die Nutzung des Internet als direkten Vertriebskanal bringt neben datenschutzrechtlichen Problemen weitere Fragen mit sich. Bei Vertragsabschlüssen im Internet sind die Anforderungen des BGB, des AGB-Gesetzes, des Versicherungsvertragsrechts und des Versicherungsaufsichtsrechts einzuhalten. Da die daraus folgenden rechtlichen Schwierigkeiten nicht einfach zu überwinden sind, nutzen nur sehr wenige Versicherungsunternehmen das Internet zum Vertragsabschlu $\beta^{9}$.

Die rechtlichen Bedenken gegen einen Vertragsabschluß auf einem virtuellen Markt werden freilich nicht in allen Ländern geteilt. Der Abschluß von Versicherungsverträgen über das Internet gehört in einigen Ländern schon zum Standard des Internet-Auftritts eines Versicherers. So nutzen ca. $50 \%$ der US-Versicherungsunternehmen das Internet, um Verträge abzuschließen ${ }^{10}$. Deutsche Versicherungsunternehmen werden sich diesem Trend nicht entziehen können, auch wenn die rechtlichen Rahmenbedingungen noch nicht in allen Einzelheiten geklärt sind.

\section{Wirtschaftliche Chancen des Internets für Versicherungsunternehmen}

Die wirtschaftlichen Rahmendaten des Internets sind für alle Unternehmen einschließlich der Versicherungsunternehmen sehr verlockend. Im Jahr 2000 sollen die Einnahmen aus dem gesamten electronic commerce (einschließlich des Internets) $200 \mathrm{Mrd}$. ECU betragen ${ }^{11}$. Internet-Nutzer sind in der Regel jüngere Menschen mit einem Durchschnittsalter von 29 bis 34 Jahren, die über eine gehobene berufliche Ausbildung und gutes Einkommen verfügen ${ }^{12}$. Gerade diese Bevölkerungsschicht plant vornehmlich in dieser Lebensphase die finanzielle Absicherung. Sie ist daher für Versicherungsunternehmen eine besonders interessante Zielgruppe.

Nur ca. 1 - $2 \%$ der im Internet präsenten Unternehmen bieten im Bereich der Rechtsschutz-, Hausratund Haftpflichtversicherung einen direkten Vertragsabschluß an; vgl. F.A.Z. Nr. 201 vom 31.8.1998, S. 28.

$10 \quad$ Hartmann, VW 1997, S. 1438 (1439).

$11 \quad \operatorname{KOM}(97) 157$ endg. , S. 9 ff.

12 Schöffski/Samusch, ZVersWiss 1997, S. 171 (186). 
Es steht $\mathrm{zu}$ erwarten, daß auch in Deutschland in Zukunft immer mehr Versicherungsunternehmen das Internet nutzen und einen Online-Vertragsabschluß anbieten werden. Dabei werden einfache oder standardisierte Produkte, wie z.B. die Privathaftpflicht-, die Kfz- oder die Reisekrankenversicherung, vor beratungsintensiven Produkten wie der Kapitallebensversicherung den Vorzug erhalten. Der Online-Vertrieb von Versicherungen könnte attraktiver ausgestaltet werden, wenn die Unternehmen für diesen Vertriebsweg spezielle Produkte entwickeln, die auf die oben beschriebene Nutzergruppe zugeschnitten sind.

Der Aufbau einer Homepage ist im Vergleich zu anderen Marketing-Instrumenten überaus preiswert $^{13}$. Für junge Versicherungsunternehmen und Branchenneulinge ist es daher möglich, mit einem relativ kleinen Kapitaleinsatz für den Online-Auftritt mit großen Unternehmen in Wettbewerb zu treten. Die Aktualisierung der Websites kann sehr schnell erfolgen, und neue Produkte können umgehend nach der Marktreife vertrieben werden. Gleichzeitig kann am kostenintensiven Vertrieb gespart werden ${ }^{14}$. Das Internet ermöglicht einen standortunabhängigen und globalen Vertrieb von Versicherungsdienstleistungen, der nicht von den üblichen Geschäfts- und Bürozeiten eingeschränkt wird.

Weitere Einsparungen können erfolgen, wenn der gesamte Vertrag einschließlich Abschluß, Bezahlung, Vertragsanpassung, Schadensmeldung und Beendigung online abgewickelt wird. Die ersparten Kosten können an den Versicherungsnehmer weitergeben werden. Für die Versicherungsunternehmen bedeuten geringere Kosten einen erheblichen Wettbewerbsvorteil, der in Zeiten zunehmenden Wettbewerbs nicht zu unterschätzen ist.

Die Deregulierung im Versicherungsrecht durch den europäischen Gesetzgeber hat dazu geführt, daß es für Unternehmen einfacher wurde, grenzüberschreitend Dienstleistungen anbieten zu können. Die Versicherer sind dadurch in der Lage, europaweit Kundengruppen anzusprechen, die sie bisher gar nicht oder die sie nur mit hohem Kostenaufwand ansprechen konnten $^{15}$. Die europäische Währungsunion und die Einführung des Euro tragen zu einer Ausweitung des grenzüberschreitenden Handels bei. Für die Verbraucher bedeutet dies eine

13 Schöffski/Samusch, ZVersWiss 1997, S. 171 (191).

14 Einige Versicherungen bieten einen 10 - 20\% Abschlag auf die Versicherungsprämie bei einem Online-Vertragsabschluß an, FAZ, vom 31.8.1998, S. 28 und Handelsblatt vom 10.2.1998.

15 Hartmann, VW 1997, S, 1438 (1440). 
erhöhte Preistransparenz bei den einzelnen Versicherungsprodukten ${ }^{16}$. Für die Versicherungsunternehmen führt dies zu einem verstärkten Wettbewerb.

Vor der Nutzung des Internets zur globalen oder europaweiten Erbringung von Dienstleistungen bedarf es aber einer genauen Prüfung, ob nicht gegebenenfalls bestehende Nachteile die Vorteile überwiegen. Um dies zu verdeutlichen, soll kurz die Situation auf EUEbene beleuchtet werden. Die Mitgliedstaaten sind berechtigt, aus Gründen des Allgemeininteresses besondere Regelungen auch für bereits liberalisierte und harmonisierte Wirtschaftszweige zu erlassen, die besonders den Markteintritt von ausländischen Unternehmen beeinträchtigen können ${ }^{17}$. Versicherungsunternehmen müssen solche Regelungen beim Abschluß eines Vertrages über das Internet beachten. Ferner sind die pflichtgemäße Bestellung eines steuerlichen Vertreters im Aufnahmestaat und eines Vertreters für örtliche Versicherungsansprüche in dem Land, in dem das Risiko belegen ist, sehr kostenintensiv ${ }^{18} . \mathrm{Zu}$ beachten ist auch, daß es Unterschiede im Versicherungsrisiko zwischen den einzelnen Staaten geben kann. Eine Bonitätsprüfung des Kunden kann bei grenzüberschreitendem Dienstleistungsverkehr kostenintensiv sein. Für Versicherungsunternehmen kann es daher einfacher sein, den Vertragsabschluß mit Kunden außerhalb des Herkunftsstaates über das Internet zu verweigern oder zu begrenzen ${ }^{19}$. Dies kann mit einem entsprechenden Hinweis auf der Homepage angekündigt werden. Die Vertragsfreiheit ermöglicht ein solches Vorgehen, wenn nicht wettbewerbsrechtliche Regelungen dem entgegenstehen.

Aber auch für den Verbraucher im Aufnahmestaat mag nicht jeder Abschluß eines Versicherungsvertrags über das Internet vorteilhaft sein. In einigen EU-Mitgliedstaaten sind die Prämien für die Lebensversicherung nicht von der Einkommensteuer abzugsfähig, wenn der

Erste Tests haben jedoch ergeben, daß es sich gegenwärtig finanziell noch nicht lohnt, grenzüberschreitend Versicherungsprodukte nachzufragen, Finanztest 12/97 S. 80.

Dazu umfassend der Entwurf einer Mitteilung der Kommission zu Auslegungsfragen, Freier Dienstleistungsverkehr und Allgemeininteresse im Versicherungswesen, CAB XV/207/97 - DE Rev. 2.

KOM 96 (209) endg.; S. 9.

Bei einem Test der Zeitschrift Finanztest 12/97, S. 80 verweigerten von 156 befragten Gesellschaften 79 den grenzüberschreitenden Vertragsschluß. 
Vertrag mit einer nicht im Aufnahmestaat ansässigen Versicherungsgesellschaft geschlossen wurde $^{20}$.

Im Ergebnis ist festzustellen, daß das Internet eine gute Ergänzung $\mathrm{zu}$ den klassischen Vertriebswegen bieten kann. Für die Zukunft ist eine erhöhte Internet-Präsenz der Versicherungsunternehmen $\mathrm{zu}$ erwarten. Bei der Entwicklung spezieller Versicherungsprodukte für den elektronischen Absatz kann es für die Versicherungsunternehmen und Verbraucher überaus lohnend sein, das Internet zum Abschluß von Versicherungsverträgen zu nutzen.

Dieser Beitrag konzentriert sich auf die rechtlichen Aspekte der Internet-Nutzung durch Versicherungsunternehmen als Anbieter. Die allgemeinen Probleme des Online-Auftritts, wie die Voraussetzungen für Hard- und Software ${ }^{21}$, werden daher ebenso ausgeklammert wie Probleme bei der Betätigung von Versicherungsunternehmen als Nachfrager im Internet, bei der unternehmensinternen Internet-Nutzung (Intranet) sowie der Bezahlung der Prämien mit dem sog. Cybergeld ${ }^{22}$.

\section{Allgemeine Rechtsprobleme bei der Internet-Nutzung}

Neben einigen versicherungsspezifischen Regelungen sind zunächst die allgemeinen Rechtsvorschriften - auch im Internet - zu beachten. In diesem Sinne ist das Internet also keineswegs ein rechtsfreier Raum. Es gelten vielmehr allgemeine sowie online-spezifische Regelungen, insbesondere Daten- und Verbraucherschutz-, Marken- und Wettbewerbsrechtsnormen sowie zivilrechtliche Bestimmungen, die hier zu Beginn dargestellt werden sollen.

20 Vgl. zur rechtlichen Zulässigkeit dieses Vorgehens EuGH, Urteil vom 28.1.1992, C-204/90, Slg. 1992, S. 249 - Bachmann.

21 Weiterführend Blawath, VW 1997, S. 518 (520); Schöffski/Samusch, ZVersWiss 1997, S. 171 (188 ff.).

22 Vgl. dazu Strömer, Onlinerecht, Heidelberg 1997, S. 115 ff. 


\section{Rechtliche Einordnung einer Unternehmens-Homepage}

Der Kontakt des Versicherungsunternehmens mit der Netzwelt vollzieht sich vor allem über die Homepage des Anbieters. Es stellt sich deshalb zunächst die Frage, welche rechtlichen Vorgaben für die Homepage an sich gelten. Was ist eine Homepage rechtlich? Um es vorwegzunehmen: eine allgemeingültige Aussage darüber läßt sich nicht treffen. Es kommt auf die Gestaltung und die Funktion der Homepage im Einzelfall an, weil das Recht der „Multimediadienste“ bzw. - in juristischer Terminologie - der Informations- und Kommunikationsdienste auf ein Bundesgesetz und einen Länderstaatsvertrag aufgeteilt wurde $^{23}$. Es sind dies das Informations- und Kommunikationsdienstegesetz des Bundes (IuKDG) und der Mediendienstestaatsvertrag (MDStV) der Länder²4.

Das Teledienstegesetz $(\mathrm{TDG}=$ Art. 1 IuKDG) erfaßt gemäß $\S 2$ Abs. 1 ,alle elektronischen Informations- und Kommunikationsdienste, die für eine individuelle Nutzung von kombinierbaren Daten wie Zeichen, Bilder oder Töne bestimmt sind und denen eine Übermittlung mittels Telekommunikation zugrunde liegt (Teledienste)““. Gemäß $§ 2$ Abs. 1 MDStV soll der Mediendienstestaatsvertrag gelten „für das Angebot und die Nutzung von an die Allgemeinheit gerichteten Informations- und Kommunikationsdiensten (Mediendienste) in Text, Ton oder Bild, die unter Benutzung elektromagnetischer Schwingungen ohne Verbindungsleitung oder längs oder mittels eines Leiters verbreitet werden“. Das TDG zielt somit auf die Erfassung der Individualkommunikation sowie der „,individualkommunikationsnahen Telekommunikation“25 $\mathrm{ab}^{26}$. Dagegen sind Mediendienste wegen des Merkmals der Adressierung an die Allgemeinheit der Massenkommunikation

Dieser Kompromiß war notwendig geworden, nachdem sowohl der Bund als auch die Länder die Gesetzgebungszuständigkeit für diese Materie beanspruchten. Siehe dazu Kröger/Moos, ZUM 1997, S. 462.

Das Problem der ausschließlichen Zuordnung eines Dienstes zu einem der beiden Gesetze und damit auch eine trennscharfe Abgrenzung der beiden Dienstformen „Teledienst“ und „Mediendienst“ hat man durch überwiegend gleichlautende Bestimmungen mit identischen Rechtsfolgen in beiden Regelwerken umgehen wollen. Da in wichtigen Einzelheiten aber Unterschiede zwischen beiden Gesetzen bestehen, kann auf eine eindeutige Zuordnung nicht verzichtet werden; vgl. auch Moos, DuD 1998, S. 162 (163).

25 Bullinger/Mestmäcker, Multimediadienste - Aufgaben und Zuständigkeit von Bund und Ländern, Rechtsgutachten 1996, S. 156.

26 Ebenso: Ring, ZUM 1998, S. 358 (360). 
zuzurechnen. Der Unterschied zwischen den beiden Dienstformen - und damit der für die Abgrenzung zwischen beiden wesentliche Aspekt - beruht auf den zwei gegenläufigen Merkmalen der „Bestimmung für eine individuelle Nutzung“ bei Telediensten und der „Adressierung an die Allgemeinheit" bei Mediendiensten ${ }^{27}$.

Die Einordnung von Homepages unter eine der beiden Kategorien wirft deshalb große Probleme auf, weil beim „Anklicken“ einer Homepage im Internet zwar technisch jeweils nur eine Kommunikationsverbindung zwischen einzelnen Beteiligten zustandekommt, grundsätzlich aber eine beliebige Vielzahl von Personen diesen Einzelabruf vornehmen kann, so daß bei Homepages sowohl Elemente der individuellen Nutzung wie auch der Adressierung an die Allgemeinheit vorliegen. Neben der technischen Übermittlungsart muß es deshalb vor allem auf die Funktion der Homepage ankommen. Wie bereits dargelegt, können sich die InternetAngebote von Versicherungsunternehmen erheblich voneinander unterscheiden. Neben rein werbenden Angeboten, die sich auf eine Unternehmens- und Produktpräsentation beschränken, sind Online-Schadensmeldungen, Prämienberechnungen, Gesundheitsprüfungen und OnlineVertragsabschlüsse denkbar.

Um die Zuordnung der Dienste im Einzelfall zu erleichtern, haben Bundes- und Landesgesetzgeber Regelbeispiele für Tele- und Mediendienste normiert. Gemäß $§ 2$ Abs. 2 Nr. 2 TDG sollen Angebote zur Information und Kommunikation, soweit nicht die redaktionelle Gestaltung im Vordergrund steht, als Teledienste einzustufen sein. Eine Unternehmens-Homepage, die vorrangig eine Darstellung des Unternehmens und der Mitarbeiter und Produktinformationen beinhaltet, dürfte deshalb als Teledienst anzusehen sein. Jedoch dürften die Übergänge zu einem redaktionell gestalteten Angebot, in Form einer Unternehmensbroschüre etwa, die auch den Abruf von Pressemitteilungen und gegebenenfalls sachbezogene Artikel enthält, fließend sein. Solche Angebote könnten gemäß § 2 Abs. 2 Nr. 4 MDStV als Bilddarbietungen auch als Mediendienste zu qualifizieren sein, da weder die reine Übermittlung von Daten noch der individuelle Leistungsaustausch im Vordergrund steht. 
Angebote, Online-Schadensmeldungen, Prämienberechnungen, Gesundheitsprüfungen und Vertragsabschlüsse durchzuführen dürfte in die Kategorie der Angebote im Bereich der Individualkommunikation gemäß $\S_{2} 2$ Abs. 2 Nr. 1 TDG fallen. Bei den online durchzuführenden Schadensmeldungen, Prämienberechnungen, Gesundheitsprüfungen und auch Vertragsabschlüssen werden ja lediglich Online-Formulare bereitgehalten, nicht aber ein inhaltliches Angebot erstellt, so daß kein Anknüpfungspunkt für eine Qualifikation als Mediendienst gegeben ist.

Das ergibt sich auch aus $\S 2$ Abs. 2 Nr. $4 \mathrm{MDStV}$, wonach Mediendienste insbesondere Abrufdienste sind, bei denen Text-, Ton- oder Bilddarbietungen auf Anforderung aus elektronischen Speichern zur Nutzung übermittelt werden, mit der Ausnahme solcher Dienste, bei denen der individuelle Leistungsaustausch oder die reine Übermittlung von Daten im Vordergrund steht, ferner von Telespielen“. Beim Online-Vertragsschluß, ggf. mit OnlineBezahlung, steht wohl der individuelle Leistungsaustausch im Vordergrund ${ }^{28}$.

Die Kombination verschiedener Angebotstypen in einem Dienst (z.B. unter einer Homepage) kann darüber hinaus zu einem sehr heterogenen Erscheinungsbild des Gesamtangebots führen. $\mathrm{Ob}$ in diesem Fall eine Beurteilung jeder einzelnen Komponente (ggf. jeder einzelnen InternetSite) oder eine Gesamtbetrachtung vorzunehmen ist, ist noch nicht geklärt. Teilweise wird vertreten, daß jedes einzelne Angebot für sich als Dienst i.S.d. Gesetze aufzufassen $\operatorname{sei}^{29}$. Der Wortlaut und die Systematik des MDStV sprechen hingegen dafür, daß ein Mediendienst sich aus (mehreren) Angeboten zusammensetzen $\mathrm{kann}^{30}$. Sinnvoll und geboten für die Einordnung solcher Dienste als Mediendienst oder Teledienst erscheint deshalb eine Gesamtbetrachtung der Angebote im Wege einer, ,wertenden Gesamtschau“31, die insbesondere den Anteil der jeweiligen Einzelangebote am Gesamtangebot berücksichtigt. Eine solche Differenzierung ist jedoch ohnehin nur dann geboten, wenn nicht alle unter einer Homepage zusammengefaßten Angebote derselben Kategorie (Teledienst oder Mediendienst) zuzuordnen sind.

28 Vgl. Kröger/Moos, AfP 1997, S. 675 (679 f.).

29 Pichler, MMR 1998, S. 79 (80); Engel-Flechsig/Maennel/Tettenborn, NJW 1997, S. 2981 (2982).

$30 \quad$ Vgl. Waldenberger, MMR 1998, S. 124 (125);

31 Waldenberger, MMR 1998, S. 124 (125). 
In der Regel werden Unternehmens-Homepages allgemein und damit auch VersicherungsHomepages als Teledienste im Sinne des TDG aufzufassen sein $^{32}$, auch wenn sie mehrere der genannten Nutzungsmöglichkeiten bieten.

\section{Anforderungen des TDG}

Damit finden grundsätzlich sämtliche Vorschriften des TDG Anwendung. Gemäß $§ 4$ TDG können solche Teledienste zulassungs- und anmeldefrei betrieben werden. Im Regelfall werden größere Unternehmen ihr Web-Angebot auf einem eigenen Server bereithalten und in das Netz einspeisen. Damit sind sie Diensteanbieter im Sinne von $\S 3$ Nr. 1 TDG. Sie sind gemäß $\S 5$ Abs. 1 TDG für die in das Internet eingestellten Inhalte nach den allgemeinen Gesetzen verantwortlich. Eine Haftungsprivilegierung gibt es nur für solche Anbieter, die fremde Inhalte zur Nutzung bereithalten und gegebenenfalls einen Haftungsausschluß, wenn zu fremden Inhalten lediglich der Zugang vermittelt wird. Dies könnte zum Beispiel der Fall sein, wenn in einer Homepage mittels eines Hyperlinks auf eine fremde Seite verwiesen wird. Ist der Verweis auf eine fremde Homepage aber nicht hinreichend deutlich und wird somit der Eindruck erweckt, die angesteuerte Seite gehöre zum eigenen Angebot (sog. Inline-Linking), kann den Anbieter die volle Haftung treffen. Die Einzelheiten in diesem Bereich sind noch nicht geklärt ${ }^{33}$.

Geschäftsmäßige Angebote - also auch Unternehmens-Homepages - müssen gemäß § 6 TDG über eine Anbieterkennzeichnung verfügen, die Namen und Anschrift des Anbieters bzw. des Vertretungsberechtigten enthält ${ }^{34}$.

\section{Schutz des Domain-Namens}

Die Homepage ist Visitenkarte und Anlaufstation für die Kunden. Schon hier liegt ein wesentlicher Schlüssel zum Erfolg des gesamten Internet-Angebots: nur wer über eine eingängige und zugleich aussagekräftige Benennung seiner Homepage (sog. Domain) verfügt,

32 Vgl. Moos, in: Gimmy/Kröger (Hrsg.), Fn. 27; Hoeren, Rechtsfragen des Internet, 1998, Rn. 251.

33 Vgl. Müller-Terpitz, Verantwortung und Haftung der Anbieter, in: Gimmy/Kröger (Hrsg.), Fn. 27;

Spindler, ZUM 1996, S. 533; Koch, CR 1997, S. 193; Eichler/Helmers/Schneider, MMR 1998, S. 23.

$34 \quad$ Weitere Informationspflichten können sich aus versicherungsspezifischen Normen ergeben, wie $\S 10$ a

VAG i.V.m. der Anlage D zum VAG ergeben. 
kann sich aus der zum Teil unübersehbaren Masse der Internet-Angebote herausheben und hat so Aussicht darauf, im Netz „gefunden“ zu werden.

Grundsätzlich sind die Domain-Namen von dem die Registrierung Beantragenden frei wählbar $^{35}$. Domains können jedoch auf unterschiedliche Weise rechtlich geschützt sein. Nach den Vergabebedingungen der DENIC sind die Antragsteller für etwaige marken- oder namensrechtlichen Folgen aus der Registrierung des Domain-Namens selbst verantwortlich. Schadensersatz und / oder Abwehr- und Unterlassungsansprüche können die Folge sein.

\section{a) Namensschutz gemäß $§ 12$ BGB}

Nach mittlerweile nahezu einhelliger Auffassung kommt Domains eine Namensfunktion im Sinne von $\S 12$ BGB zu, da Domains über die Aufgabe der technischen Vermittlung hinaus auch eine Kennzeichnungsfunktion besitzen ${ }^{36}$. Gemäß $\S 12$ BGB genießen auch juristische Personen Namensschutz, z. B. bezüglich der Firma, vor dem unbefugten Gebrauch des Namens, etwa als Domain-Name. Bei der Wahl der eigenen Domain sollte deshalb darauf geachtet werden, daß keine Namensrechte Dritter verletzt werden. Werden eigene Namensrechte dadurch verletzt, daß sich ein Dritter eine Domain unter dem geschützten Namen registrieren läßt, besitzt der Namensinhaber aus $\S 12$ BGB einen Beseitigungs- und bei Wiederholungsgefahr - einen Unterlassungsanspruch.

\section{b) Schutz als geschäftliche Bezeichnung gemäß $§$ MarkenG}

Zusätzlich können Domains als Unternehmenskennzeichen Rechtsschutz durch $\S 5$ Abs. 1 und 2 MarkenG genießen. Zwar wird, parallel zu der Diskussion im Rahmen von $§ 12$ BGB, den Domains eine auch hier erforderliche Kennzeichnungsfunktion teilweise abgesprochen ${ }^{37}$; diese Ansicht dürfte aber nicht mehr haltbar sein. Das läßt sich insbesondere mit der wachsenden Bedeutung der Domains als Marketinginstrument begründen ${ }^{38}$. Zahlreiche Unternehmen

In Deutschland ist für die Vergabe und die technische Einrichtung von Domains unter dem Top-Level .de die DENIC am Rechenzentrum der Universität Karlsruhe (http://www.nic.de) zuständig. Zur Praxis der Domain-Vergabe s. Hoeren (Fn 32), Rn. 47 ff. CR 1997, S. 291 f. und 377 f.

$37 \quad$ Vgl. Kur, CR 1996, S. 327.

$38 \quad$ Hoeren (Fn 32), Rn. 52. 
werben mittlerweile auch außerhalb des Internets in anderen Medien mit ihrer InternetAdresse. Diese stellt einen immer bedeutender werdenden, eigenständigen Teil der Corporate Identity des Unternehmens dar.

Gemäß $§ \quad 15$ Abs. 2 i. V. m. Abs. 4 und 5 MarkenG hat der Inhaber des Unternehmenskennzeichens das Recht, den unbefugten Benutzer des Kennzeichens - z. B. als Domain - auf Unterlassung und gegebenenfalls auf Schadensersatz in Anspruch zu nehmen. Ein solcher Anspruch besteht auch dann, wenn nur ein ähnliches Kennzeichen verwendet wird, durch das die Unterscheidungskraft oder die Wertschätzung des Unternehmenskennzeichens in unlauterer Weise ausgenutzt oder beeinträchtigt wird ( $§ 15$ Abs. 3 MarkenG) ${ }^{39}$.

Ein solches Vorgehen ist auch gegen vermeintliche Privatpersonen möglich, die oft mehrere Firmennamen als Domains auf sich registrieren lassen (sog. „Domain-Grabbing“). Zwar setzt $\S 5$ Abs. 2 MarkenG die „Bedeutung des Kennzeichens im geschäftlichen Verkehr“ voraus, was den rein privaten Gebrauch nicht umfaßt. Dieses Merkmal soll nach der Rechtsprechung jedoch bereits dann erfüllt sein, wenn sich Private zum Zwecke des Weiterverkaufs entsprechende Domains reservieren lassen ${ }^{40}$.

\section{c) Benutzung von Gattungsbegriffen}

In der Regel werden sich etablierte Versicherungsunternehmen ihren bekannten und eingeführten Firmennamen sinnvollerweise auch als Domain registrieren lassen. Bei neu auf den Markt tretenden Versicherern oder solchen, die bewußt ein eigenständiges Internet-Angebot aufbauen wollen, ist eine solche Identifikationsfunktion der Domain unter Umständen nicht in gleichem Maße notwendig. Um einen möglichst großen Zulauf im Internet zu erhalten, könnte sich die Verwendung eines Gattungsbegriffs als Domain, z. B. „versicherung.de“, empfehlen. Die Verwendung solcher Domains ist kennzeichenrechtlich grundsätzlich zulässig ${ }^{41}$, wenn dem nicht wettbewerbsrechtliche Bedenken entgegenstehen ${ }^{42}$.

\footnotetext{
39 Siehe dazu im einzelnen: Jaeger-Lenz, MMR 1998, S. 9 (12 ff.).

Vgl. LG Düsseldorf, WM 1997, S. 1444; LG Frankfurt, CR 1997, S. 287; LG Lüneburg, CR 1997, S. 288.

$41 \quad$ OLG Frankfurt, Beschluß v. 13.2.1997, 6 W 5/97 - nicht rechtskräftig.

$42 \quad$ Vgl. hierzu Hoeren (Fn 32), Rn. 61 f.
} 


\section{Werbung / Wettbewerbsrecht}

Es wurde bereits darauf hingewiesen, daß die meisten Versicherungsunternehmen das Internet gegenwärtig als Werbeträger nutzen. Grundsätzlich haben Versicherungsunternehmen das Recht, uneingeschränkt - auch europaweit ${ }^{43}$ - für ihre Dienstleistungen zu werben. Die Einzelheiten der Zulässigkeit verschiedener Werbeformen im Internet sind aber noch nicht vollständig ausgelotet. Unklar ist bislang, ob für Homepages das sog. Trennungsgebot gelten soll, demzufolge Werbung klar erkennbar vom übrigen Inhalt getrennt werden muß ${ }^{44}$. Das Teledienstegesetz enthält diesbezüglich keine Regelung. Der MDStV hingegen normiert ein solches Trennungsgebot in $\S 9$ Abs. 2 MDStV. Bei Zugrundelegung der oben vorgenommenen Regelqualifizierung von Unternehmens-Homepages als Teledienst wäre eine Trennung mithin nicht erforderlich. Auf Werbung im Internet findet im übrigen das allgemeine Wettbewerbsrecht, also vor allem $\S \S 1$ und 3 UWG Anwendung ${ }^{45}$. Ein Trennungsgebot könnte sich mithin auch für Teledienste aus diesen Vorschriften ergeben ${ }^{46}$. Dagegen dürfte jedoch die Erwartungshaltung der Nutzer sprechen: auf Unternehmens-Homepages wird man keine marketingunabhängigen, objektiven Informationen erwarten können ${ }^{47}$.

Ein wettbewerbsrechtlich relevantes Verhalten kann zudem in der Verwendung von Hyperlinks bestehen. So dürfte $\S 1$ UWG der unlauteren Übernahme einer fremden Homepage in das eigene Angebot (mittels sog. Inline-Linking) entgegenstehen ${ }^{48}$. Aber auch schon der ganz

Vgl. Art. 41 Richtlinie 92/96/EWG des Rates v. 10.11 .1992 zur Koordinierung der Rechts- und Verwaltungsvorschriften für die Direktversicherung (Lebensversicherung) sowie zur Änderung der Richtlinien 79/267/EWG und 90/619/EWG (Dritte Richtlinie Lebensversicherung), AB1. EG Nr. L 360 v. 9.12.1992, S. 1 (21); Art. 41 Richtlinie 92/49/EWG des Rates vom 18.6.1992 zur Koordinierung der Rechts- und Verwaltungsvorschriften für die Direktversicherung (mit Ausnahme der Lebensversicherung) sowie zur Änderung der Richtlinien 73/239/EWG und 88/357/EWG (Dritte Richtlinie Schadenversicherung), ABl. EG Nr. L 228 v. 11.8.1992, S. 1 (20). Zur Einordnung von Homepages als Werbung s. Marwitz, MMR 1998, S. 188.

45 Zum Verbot vergleichender Tarifberechnung im Internet OLG Frankfurt, Az.: 6 U 217/97. Für einige Berufe gelten im übrigen besondere standes- und berufsrechtliche Beschränkungen, z.B. für Rechtsanwälte und Ärzte, vgl. dazu Strömer (Fn. 22), S. 110 ff. 
reguläre Verweis auf ein konkurrierendes Unternehmen kann wettbewerbsrechtlich zu beanstanden $\operatorname{sein}^{49}$.

Weitgehend einhellig scheint mittlerweile die Frage nach der Zulässigkeit unaufgefordert verschickter E-Mail-Werbung beurteilt zu werden. In Anlehnung an die Rechtsprechung zu unverlangt zugesandter Telefax- und Btx-Werbung wird auch in E-Mails eine gegen $\S 1$ UWG verstoßende Belästigung des Adressaten gesehen ${ }^{50}$.

\section{Datenschutz}

Im Rahmen ihrer gewöhnlichen Geschäftstätigkeit haben Versicherungsunternehmen generell die allgemeinen Datenschutzgesetze - also insbesondere das Bundesdatenschutzgesetz (BDSG) - zu beachten. Das gilt auch dann, wenn sie für ihre Tätigkeit im Internet werben oder ihre Verträge über dieses Medium abschließen. Aufgrund der hier kurz darzustellenden besonderen Datenschutzrisiken öffentlicher Kommunikationsnetze bestehen aber darüber hinaus Spezialvorschriften, die im Rahmen der Internet-Tätigkeit zusätzlich zu beachten sind. Unberührt davon bleiben die datenschutzrechtlichen Pflichten im Rahmen des gewöhnlichen Versicherungsgeschäfts ${ }^{51}$.

Bei der Internet-Kommunikation werden neben den unverschlüsselten Inhaltsdaten mit jedem Datenpaket auch zwangsläufig Informationen über die Identität von Sender und Empfänger (Kennung des Rechners), den Zeitpunkt der Sendung und den Übertragungsweg übertragen. Diese "Datenspur" kann an jedem Server, den das Datenpaket passiert, abgehört, zurückverfolgt, verändert, gefälscht und unterdrückt werden. Das Kommunikationsnetz stellt gleichzeitig die Infrastruktur zur Verfügung, die so gewonnenen Daten auf beliebige andere an das Netzwerk angeschlossenen Computersysteme zu übertragen, so daß grundsätzlich an

$49 \quad \mathrm{Zu}$ den Einzelheiten vgl. Hoeren (Fn 32), Rn. 266 ff.

50 LG Traunstein, NJW-CoR 1997, S. 494; Dethloff, NJW 1998, S. 1596 (1598); Schmittmann, DuD 1997, S. 636 (639); Ernst, BB 1997, S. 1057 (1060); Schrey/Westerwelle, BB 1997, Beil. 18, S. 17 (20 ff.); Gummig, ZUM 1996, S. 573; Hoeren, in: Schriftenreihe des Instituts für Rundfunkrecht an der Universität zu Köln, Band 71, Die Zukunft der Medien hat schon begonnen - Rechtlicher Rahmen und neue Teledienste im Digitalzeitalter, München 1998, S. 57 (65 f.); a.A.: Reichelsdorfer, GRUR 1997, S. 191 (197).

51 Dazu Schmidt, Die Datenverarbeitung im Versicherungsbetrieb, Wiesbaden 1993, S. 64 f. 
jedem Ort mit Netzzugang diese Protokolldateien ausgewertet und Benutzungsprofile erstellt werden können. Insbesondere in Privathaushalten, in denen in der Regel nur ein Einzelrechner vorhanden ist, sind die Rechneradressen unmittelbar natürlichen Personen zugeordnet, so daß diese anonymen Nutzungsprofile leicht zu Nutzerprofilen werden können.

Die Nutzer von über das Internet angebotenen Telediensten haben deshalb ein besonderes Schutzbedürfnis, welches jedoch innerhalb der privatwirtschaftlich ausgestalteten Kommunikationsbeziehungen mit dem berechtigten Interesse der Diensteanbieter, wirtschaftlich nutzbringende Daten frei erheben und verarbeiten $\mathrm{zu}$ dürfen, zum Ausgleich gebracht werden muß. Dieses Ziel verfolgt das Teledienstedatenschutzgesetz (TDDSG) ${ }^{52}$.

In $\S 3$ TDDSG sind die Grundsätze für die Verarbeitung personenbezogener Daten bei Telediensten aufgenommen. In $\S 3$ Abs. 1 TDDSG wird in Anlehnung an $\S 4$ Abs.1 BDSG für die Erhebung, Verarbeitung und Nutzung personenbezogener Daten durch die Anbieter von Telediensten ein für das Datenschutzrecht charakteristisches Verbot mit Erlaubnisvorbehalt normiert. Eine Erlaubnis zur Datenerhebung-, verarbeitung und -nutzung liegt dann vor, wenn es eine gesetzliche Vorschrift ausdrücklich vorsieht oder der Nutzer selbst eingewilligt hat.

Das TDDSG gestattet die Erhebung, Verarbeitung und Nutzung personenbezogener Daten in $\S 5$ TDDSG für sogenannte Bestandsdaten und in $\S 6$ TDDSG für sogenannte Nutzungs- und Abrechnungsdaten. Bestandsdaten sind solche zur Begründung, Ausgestaltung und Änderung des Vertragsverhältnisses ${ }^{53}$. Da Unternehmens-Homepages regelmäßig kostenfrei und ohne vertragliche Abreden von jedermann angesteuert und das bereitgehaltene Angebot genutzt werden kann, wird ein Vertrag über die Nutzung eines Teledienstes in dieser Beziehung in der Regel nicht geschlossen. Etwas anderes könnte jedoch gelten, wenn das Unternehmen eine Newsgroup oder eine Mailingliste unterhält, in deren Rahmen regelmäßig Mitteilungen - ggf. gegen Entgelt - an Kunden versandt werden. Über den Erlaubnisvorbehalt und die enge

52 Siehe dazu die ausführliche Darstellung von Moos; Datenschutz im Internet, in: Gimmy/Kröger (Hrsg.) Fn 27; Schrader, CR 1997, S. 707; Engel-Flechsig, RDV 1997, S. 59; Gola/Müthlein, RDV 1997, S. 193

53 Freilich geht es hier um den Vertrag über die Nutzung des Teledienstes selbst, nicht etwa um einen im Rahmen der Inanspruchnahme eines Teledienstes geschlossenen weiteren Vertrag (etwa einen online geschlossenen Versicherungsvertrag). 
Bindung an den Vertragszweck hinaus enthält das TDDSG keine weiteren Beschränkungen für die Verarbeitung von Bestandsdaten, die sich im übrigen nach dem allgemeinen Datenschutzrecht, also dem BDSG, richtet.

$\S 6$ TDDSG erlaubt weiterhin die Erhebung, Verarbeitung und Nutzung von Nutzungsdaten, also solcher personenbezogenen Daten, die für die Ermöglichung der Inanspruchnahme von Telediensten erforderlich sind. Darunter sind alle Informationen zur Identifikation des Nutzers oder seines Pseudonyms, Passwörter, Zugangscodes etc. zu verstehen. Des weiteren dürfen Daten, die für die Abrechnung der Inanspruchnahme von Telediensten erforderlich sind (Abrechnungsdaten) erhoben werden. Alle anderen personenbezogenen Daten der Nutzer dürfen nur mit ausdrücklicher Einwilligung erhoben werden, die gemäß $§ 3$ Abs. 7 TDDSG unter bestimmten Voraussetzungen auch elektronisch, also per Mausklick, abgegeben werden kann.

Neben diesen Erlaubnistatbeständen normiert das TDDSD noch die Grundsätze des Systemdatenschutzes und der Datenvermeidung, die als Zielvorgaben an die Diensteanbieter gerichtet sind. Ferner sind ein Auskunftsrecht des Nutzers und Bestimmungen zur Datenschutzaufsicht enthalten.

Im Regelfall werden die von Unternehmen im Internet angebotenen Teledienste, also vor allem die Homepage, nicht kommerziell angeboten werden. Es wird entweder eine rein informatorische Werbeplattform oder ein Forum für die Anbahnung anderweitiger Vertragsbeziehungen - wie für Versicherungsverträge - eröffnet, die aber selbst keine Teledienste darstellen und somit auch nicht den Datenschutzregeln des TDDSG unterliegen. Bestands- und Abrechnungsdaten werden deshalb hier in der Regel nicht anfallen. Personenbezogene (und nur solche sind von den Datenschutzvorschriften erfaßt) Nutzungsdaten werden ebenfalls erst dann erhoben werden können, wenn der Nutzer seine Identität, z.B. durch Ausfüllen eines Formulars preisgegeben hat. Anonyme Nutzungsdaten können beliebig erhoben und ausgewertet werden.

Im Ergebnis haben Versicherungsunternehmen im Rahmen eines online abgeschlossenen Versicherungsvertrages also im Regelfall - wie im Rahmen ihrer herkömmlichen Geschäftstätigkeit auch - die Regeln des BDSG einzuhalten. 


\section{Verschlüsselung}

Das bisweilen größte Hindernis einer vollständigen „Virtualisierung“ des Versicherungsgeschäfts dürfte in den Sicherheitsproblemen bei der Datenübermittlung über das Internet liegen $^{54}$. Als wichtigste Instrumente zur Sicherung elektronischer Kommunikation sind kryptographische Verfahren anzusehen, deren Haupteinsatzfelder die Verschlüsselung und die digitale Signatur sind.

Insbesondere beim Abschluß von Versicherungsverträgen werden personenbezogene Daten, wie z.B. Gesundheitsdaten bei einer Lebensversicherung, erhoben, die vertraulich zu behandeln sind. Der Versicherungsnehmer hat deshalb ein berechtigtes Interesse an einer abhörsicheren Übertragung dieser Daten zum Versicherungsunternehmen. Wie jedoch oben im Rahmen des Datenschutzes erörtert, ist die Internet-Kommunikation höchst unsicher. Deshalb sollten in jedem Fall Verschlüsselungsverfahren eingesetzt werden. Verschlüsselung dient der Gewährleistung der Vertraulichkeit elektronischer Kommunikation. In Deutschland wie in den meisten Staaten ist der Gebrauch von Verschlüsselungssoftware nicht reglementiert ${ }^{55}$. Lediglich für den Export von Verschlüsselungstechnologien bestehen Beschränkungen nach der EG-Dual-Use-Verordnung ${ }^{56}$. Danach ist für die Ausfuhr von Verschlüsselungshard- und software gemäß Art. 3 Abs. 1 VO i.V.m. Anhang I und IV der Liste der Dual-Use-Güter ${ }^{57}$ grundsätzlich eine Genehmigung erforderlich. Davon ausgenommen ist allerdings Software, die im Handel frei erhältlich ist, und Public-Domain-Software.

Die Debatte über den Zugriff staatlicher Stellen auf Schlüssel, um aus Sicherheitsinteressen jede elektronische Nachricht entschlüsseln zu können, dauert an. In der sich abzeichnenden

$54 \quad$ Vgl. Blawath, VW 1997, S. 518 (521).

55 Als einziger EG-Mitgliedstaat hat Frankreich die Kryptographie umfassend reglementiert; siehe dazu Rihaczek, DuD 1996, S. 484; keine generellen Beschränkungen bestehen in sämtlichen OECDMitgliedstaaten; siehe auch dazu die Internet Site der „Global Internet Liberty Campaign“, http://www.gilc.org/crypto/crypto-survey.html.

56 Verordnung 3381/94/EG des Rates v. 19.12 .1994 über eine Gemeinschaftsregelung der Ausfuhrkontrolle von Gütern mit doppeltem Verwendungszweck; ABl. EG Nr. L 367 v. 31.12.1994, S. 1. 
europäischen Rechtsetzung zum Gebrauch von Verschlüsselungssoftware deutete sich jedoch eine liberale Regelung an ${ }^{58}$.

\section{Abschluß von Versicherungsverträgen im Internet}

Der wirksame Abschluß von Versicherungsverträgen, der mit offline-Abschlüssen vergleichbar ist, stellt vielfältige Anforderungen an die Gestaltung des Internet-Auftritts von Versicherungen. Die allgemeinen Anforderungen, die zu einem wirksamen Vertragsabschluß erfüllt werden müssen, und die Besonderheiten des Versicherungsvertragsgesetzes (VVG) sind zu beachten.

\section{Elektronischer Vertragsschluß im Internet}

Auch für den elektronischen Vertragsabschluß im Internet gelten die allgemein bekannten Regelungen. Es ist aber jeweils zu prüfen, wie sich dieses neue Medium in die Systematik des BGB oder des Versicherungsvertragsrechts einordnen läßt. Fest steht zunächst, daß ein Vertrag, auch ein solcher über Versicherungsdienstleistungen, rechtsgültig geschlossen werden kann. Ein Versicherungsvertrag ist gesetzlich an keine Form gebunden und wird nach allgemeinem bürgerlichem Recht geschlossen ${ }^{59}$. Jede Art von Willenserklärungen - auch die elektronisch übermittelte - ist grundsätzlich rechtswirksam. Die fehlende Schriftform eines elektronisch abgeschlossenen Versicherungsvertrages steht seiner Rechtswirksamkeit somit nicht entgegen ${ }^{60}$. Auch der Versicherungsschein, für dessen Gültigkeit ein Faksimile der eigenhändigen Unterschrift gemäß $\S 3$ Abs. 1 S. 2 VVG ausreicht, könnte über das Internet zugestellt werden ${ }^{61}$.

Darauf deutet die Mitteilung der Kommission über Sicherheit und Vertrauen in elektronische Kommunikation - ein europäischer Rahmen für digitale Signaturen und Verschlüsselung, KOM (97) 503 endg. v. 8.10.1997 hin, in der ausdrücklich dafür eingetreten wird, weder eine Reglementierung des Gebrauchs der Verschlüsselung noch eine Zugriffsmöglichkeit des Staates auf Schlüssel vorzunehmen, sondern „den Gebrauch der Kryptographie zur Sicherstellung des Datenschutzes und der Vertraulichkeit nicht [zu] beschränken“ (S. 17).

$59 \quad$ Weyers, Versicherungsvertragsrecht, 2. Auflage, 1995, Rn. 166.

60 Vgl. Schmittmann, DuD 1997, S. 636 (637); anders ist dies bei Verträgen, für die gesetzlich Schriftform verbindlich vorgeschrieben ist, wie z.B. Verbraucherkreditverträge ( $\$ 4$ VerbrKrG).

61 Gleiches gilt für Mahnschreiben gemäß § 39 Abs. 1 VVG und Prämienrechnungen gemäß $§ 42$ Nr. 4 VVG; vgl. auch Bizer, in: Kröger (Hrsg.), Internet für Rechtsanwälte und Notare, S. 148 (152 f.). 
Für den elektronischen Vertragsschluß sind zwei Möglichkeiten gegeben. Verträge können durch den Austausch von elektronischen Willenserklärungen per E-Mail abgeschlossen werden. Für den Bereich der Versicherungsverträge ist es aber näherliegend, davon auszugehen, daß der Kunde das elektronische Formular auf der Web-Seite der Versicherung ausfüllt und an diese per Mausklick abschickt.

\section{a) Die Homepage als Angebot oder als invitatio ad offerendum}

Auch ein Vertragsschluß im Internet erfordert die Abgabe zweier sich deckender Willenserklärungen. Die Internet-Präsenz von Versicherungsunternehmen könnte als bloße Aufforderungen an einen anderen, ein Angebot abzugeben (unverbindliche invitatio ad offerendum) oder als ein Vertragsangebot gemäß $§ 145$ BGB angesehen werden. Bei einigen Online-Angeboten ist tatsächlich von einem verbindlichen Angebot auszugehen. So können Software-Unternehmen ihre Produkte über das Internet an jedermann durch ein download veräußern $^{62}$. Versicherungsunternehmen wollen aber nicht mit jedermann kontrahieren, sondern vor Vertragsabschluß eventuell das Risiko oder die Bonität des zukünftigen Versicherungsnehmers prüfen. Sie bedürfen daher des Schutzes der invitatio ad offerendum, die den Verkäufer vor einer unbegrenzten Anzahl von Vertragsabschlüssen und eventueller Schadensersatzforderungen bewahren will. Das Internet-Angebot ist folglich als lediglich unverbindliche invitatio ad offerendum zu bewerten ${ }^{63}$. Das Angebot zum Abschluß eines Versicherungsvertrages geht somit vom Versicherungsnehmer aus. Die Rechtslage unterscheidet sich nicht von Vertragsangeboten, die außerhalb des virtuellen Marktes vorgenommen werden ${ }^{64}$.

\section{b) Angebot und Annahme unter Abwesenden oder Anwesenden}

Geht das Angebot zum Vertragsabschluß vom Versicherungsnehmer aus, so ist zu prüfen, wann diese Willenserklärung beim Versicherungsunternehmen zugeht. Das BGB unterscheidet Willenserklärungen zwischen An- und Abwesenden. Nach der Vernehmenstheorie geht eine

Ernst, NJW-CoR 1997, S. 165 (165) Gimmy, Vertragsschluß im Internet nach deutschem Recht, in: Gimmy/Kröger, aaO Fn. 27. kritisch Mehrings, MMR 1998, S. 30 (32). 
Willenserklärung unter Anwesenden im Zeitpunkt der akustisch richtigen Vernehmung $\mathrm{zu}^{65}$. Willenserklärungen unter Anwesenden können vorliegen, wenn das Internet lediglich als Kommunikationsmedium - ähnlich wie ein Fernsprecher gemäß $§ 147$ Abs. 1 Satz 2 BGB zwischen zwei Personen genutzt wird ${ }^{66}$. Bei der Nutzung des Internet wird das Versicherungsunternehmen aber eine Datenverarbeitungsanlage oder einen elektronischen Briefkasten installiert haben. Es fehlt daher an einer Erklärung von Person zu Person, wie sie für Willenserklärungen unter Anwesenden vorausgesetzt wird. Es liegt daher eine Willenserklärung unter Abwesenden vor.

Bei einer Willenserklärung unter Abwesenden ( $\$ 130$ Abs. 1 BGB) ist der Zugang der Willenserklärung gegeben, wenn diese so in den Machtbereich des Empfängers gekommen ist, daß dieser von der Willenserklärung Kenntnis nehmen kann und unter normalen Umständen mit einer Kenntnisnahme zu rechnen ist ${ }^{67}$. Die E-Mails zum Vertragsabschluß können bei dem Versicherungsunternehmen rund um die Uhr eintreffen. Mit dem Passieren der Schnittstelle beim Versicherungsunternehmen ist das Angebot in den Machtbereich des Empfänger gelangt. Der Zugang hängt aber auch davon ab, wann mit der Kenntnisnahme zu rechnen ist. Halten Unternehmen elektronische Einrichtungen zum Empfang von Willenserklärungen vor, so müssen diese auch regelmäßig kontrolliert werden, ob Erklärungen angekommen sind. Der elektronische Briefkasten ist daher mindestens einmal täglich $\mathrm{zu}$ leeren ${ }^{68}$. Werden die Erklärungen jedoch in einem automatisierten Verfahren bearbeitet, so ist Zugang gegeben, wenn sie beim Versicherungsunternehmen eingegangen sind. Dieser erfolgt nur Bruchteile von Sekunden nach Abschicken der Nachricht durch den Versicherungsnehmer. Ein Widerruf der Willenserklärung, der gemäß $\S 130$ Abs. 2 BGB bis zum Zugang möglich ist, ist daher praktisch ausgeschlossen ${ }^{69}$. Der Versicherungsnehmer ist dann an seinen Antrag gemäß $\S 145$ BGB gebunden. Im online-Vertragsformular kann ein bestimmter Zeitraum angegeben

\footnotetext{
65 MünchKomm/Fröschler, BGB, Band I, 3. Aufl., München $1993 \S 130$ Rn. 3 und 20.

$66 \quad$ Ernst, NJW-CoR 1997, S. 165 (166); Heun, CR 1994, S. 595 (597).

BGHZ 67, 271 (275); BGH NJW 1983, S. 929 (930); Palandt/Heinrichs, BGB, 57. Aufl. München 1998, § 130 Rn. 5.

68 Heun, CR 1994, S. 595 (599); Ernst, NJW-CoR 1997, S. 165 (166); Strömer, (Fn 22), S. 90. 
werden, während dessen der Versicherungsnehmer an seinen Antrag gebunden ist ${ }^{70}$. In der Feuerversicherung sind dies gemäß der gesetzlichen Regelung des $\S 81$ Abs. 1 Satz 1 VVG zwei Wochen.

\section{c) Einbeziehung von Allgemeinen Versicherungsbedingungen als AGB}

Allgemeine Versicherungsbedingungen (AVB) stellen AGB im Sinne des AGBG dar. Infolge des Wegfalls der aufsichtsbehördlichen Genehmigung der AVB gelten gemäß $§ 23$ Abs. 3 AGBG die allgemeinen Voraussetzungen des AGBG. Die AVB müssen daher den inhaltlichen Anforderungen dieses Gesetzes genügen und wirksam in den Vertrag gemäß § 2 AGBG einbezogen werden. Dies ist aber gemäß $\S 2$ Abs. 1 AGBG nur der Fall, wenn auf die Geschäftsbedingungen im Zeitpunkt des Vertragsschlusses ausdrücklich hingewiesen wird und für den Verbraucher die zumutbare Möglichkeit der Kenntnisnahme gegeben ist.

Die zumutbare Möglichkeit der Kenntnisnahme wird von Teilen der Literatur und Rechtsprechung im elektronischen Geschäftsverkehr verneint. Es wird ausgeführt, daß das Lesen längerer Bedingungen aufgrund der langen Übertragungsdauer unzumutbar sei. Die untergerichtliche Rechtsprechung hat dies für Fälle zur Einbeziehung Allgemeiner Geschäftsbedingungen bei dem Vertragsabschluß mittels Bildschirmtext klargestellt ${ }^{71}$. Ferner wird in der nachträglichen Abänderbarkeit der elektronischen AGB durch den Verwender eine zu große Gefahr für den Verbraucher gesehen ${ }^{72}$.

Das letzte Argument kann aber nicht überzeugen. Auch beim Offline-Abschluß von Verträgen kann der Verwender nach Vertragsabschluß die AGB abändern. Da ein solcher Vorgang den Tatbestand des $\S 263$ StGB erfüllen kann, muß der strafrechtliche Schutz genügen. Hinzu kommt, daß der Verbraucher sich die AGB ausdrucken lassen kann.

Im Gegensatz zur Btx-Technik, zu der die o.g. Entscheidungen ergangen sind, ist die Übertragungsgeschwindigkeit im Internet sehr hoch. Die fortschreitende Schnelligkeit der 773 (775).

71 LG Freiburg, CR 1992; 93; LG Aachen, CR 1991, S. 222 (222 f); Ulmer/Brandsen/Hensen, AGBG, 8. Aufl. , Köln 1998, § 2 Rn. 49.

72 Wolf/Horn/Lindbacher, AGBG, 3. Aufl., München 1994, § 2 Rn. 24 
Datennetze, Rechner und Übertragungseinrichtungen führt $\mathrm{zu}$ einer sehr kurzen Übertragungsdauer der AGB. Die Kosten der Übertragung für den Verbraucher sind daher sehr gering. Der Verbraucher kann ferner die AGB auf seinem Rechner speichern oder ausdrucken. Die von der Rechtsprechung geäußerten Bedenken zur Einbeziehung von AGB in Btx-Verträgen sind daher nicht auf den Internet-Vertragsschluß zu übertragen ${ }^{73}$.

Die AGB im Internet müssen aber graphisch so dargestellt sein, daß sie leicht zu lesen und übersichtlich gestaltet sind. Bei längeren AGB kann es sich empfehlen, mittels eines gut lesbaren Links auf dem elektronischen Bestellformular den Verbraucher auf eine andere WebSeite zum Lesen der AGB zu lenken ${ }^{74}$. Nach dem AGBG können die AVB auch ohne körperliche Aushändigung ${ }^{75}$ wirksam in den Vertrag einbezogen werden, wenn sich nicht aus den Regelungen des Versicherungsvertragsgesetzes etwas anderes ergibt.

\section{d) Verbraucherinformationen}

Infolge des Wegfalls der aufsichtsrechtlichen Vorabgenehmigung der AVB durch das Dritte Durchführungsgesetz / EWG zum $\mathrm{VAG}^{76}$ ist es notwendig geworden, den Verbraucher vor Abschluß des Vertrages und während dessen Laufzeit über die das Versicherungsverhältnis prägenden Umstände umfassend $\mathrm{zu}$ informieren. Mit dieser in $\S 10$ a VAG festgelegten Informationspflicht soll der Versicherungsnehmer das unsichtbare Produkt Versicherung vor der vertraglichen Festlegung kennenlernen ${ }^{77}$. Die Versicherungsunternehmen sind verpflichtet, den Versicherungsnehmer, vor und während der Laufzeit über die wesentlichen Vertragsaspekte zu unterrichten ${ }^{78}$. Zu diesen Verbraucherinformationen zählen auch die AVB. Das VAG legt den Versicherungsunternehmen die Pflicht zur Verbraucherinformation aber nur auf, wenn der Versicherungsnehmer eine natürliche Person ist ( $\$ 10$ a Abs. 1 Satz 1 VAG). Bei

Hoeren (Fn. 27), Rn. 322; Koehler, MMR 1998, S. 289 (292); Gimmy, Vertragsschluß im Internet nach deutschem Recht, in: Gimmy/Kröger Fn. 27; Ernst, NJW-CoR 1997, S. 165 (167) .

Ernst, NJW-CoR 1997, S. 165 (167); Koehler, MMR 1998, S. 289 (291); Gimmy, Vertragsschluß im Internet nach deutschem Recht, in: Gimmy/Kröger Fn. 27.

75 Anders vgl. Lorenz, VersR 1995, S. 616 (617) mwN.

76 BGB1 1994 I, S.1630.

77 Lorenz, ZVersWiss 1995, S. 103 (106); Schimikowski, r+s 1996, S. 1 (1).

78 Zum Inhalt der Verbraucherinformationen vgl. Anlage Teil D zum VAG. Eine Erläuterung findet sich bei Schimikowski, r+s 1996, S. 1 (1 ff.). 
juristischen Personen besteht daher keine Pflicht zur Vorabinformation. Bei Großrisiken gilt nur eine beschränkte Verbraucherinformation (§ 10 a Abs. 1 Satz 2 VAG).

Diese Informationen müssen gemäß $\S 10$ a Abs. 2 VAG eindeutig formuliert, übersichtlich gegliedert und verständlich in deutscher Sprache oder der Muttersprache des Versicherungsnehmers gefaßt sein. Überdies ist die Verbraucherinformation schriftlich zu erteilen $^{79}$. Die Schriftlichkeit verlangt hier die körperliche Aushändigung an den Versicherungsnehmer ${ }^{80}$. Das Schriftlichkeitserfordernis wird deshalb von einer Webseite im Internet nicht erfüllt. Aus Gründen des Verbraucherschutzes kann auch nicht zu Ungunsten des Versicherungsnehmers vom Schriftformerfordernis abgewichen werden. Das Versicherungsunternehmen kann somit durch ein online abrufbares Dokument seiner Informationspflicht nicht nachkommen ${ }^{81}$.

Die Verletzung der in $\S 10$ a VAG aufgestellten Informationspflicht wird im VAG nicht zivilrechtlich sanktioniert. Die Aufsichtsbehörde kann aber im Rahmen der Mißstandsaufsicht gemäß $\S 81$ VAG vorgehen ${ }^{82}$.

Wenn Versicherungsunternehmen im Internet die Vorabinformation des $\S 10$ a VAG nicht erfüllen können, so ist $\mathrm{zu}$ prüfen, welche zivilrechtlichen Auswirkungen dies auf den Versicherungsvertrag hat. $\S 5$ a VVG nimmt auf die in $\S 10$ a VAG geregelte Vorabinformation Bezug und transformiert diese ins Versicherungsvertragsrecht. Mit $\S 5$ a VVG wurde ein neues Modell des Abschlusses von Versicherungsverträgen entwickelt ${ }^{83}$.

79 Vgl. auch den Anhang II der Richtlinie 92/29/EWG, ABl. EG Nr. L 360 v. 9.12.1992, S. 1 (26 f.).

$80 \quad$ Schimikowski, r+s 1996, S. 1 (1); VerBAV 95, S. 283 (283 f.).

81 Technisch wäre es aber ohne weiteres möglich, den Versicherungsnehmer die genannten Informationen vor Abgabe seiner Willenserklärung zukommen zu lassen. Dies könnte mit den oben beschriebenen Mechanismen zur Einbeziehung der AVB in den Vertrag erfolgen.

82 Lorenz, ZVersWiss 1995, S. 103 (107); Schimikowski, r+s 1996, S. 1 (2).

$83 \S 5$ a VVG ist eine Norm, die in den vergangenen Jahren oftmals Gegenstand juristischer Abhandlungen war. Es ist nicht möglich, hier auf alle rechtlichen Probleme einzugehen. Es sei daher auf das vielfältige Schrifttum verwiesen. vgl. Lorenz, VersR 1997, S. 773 ff. mwN. 


\section{aa) Antragsmodell}

Das herkömmliche Modell zum Abschluß von Versicherungsverträgen wird Antragsmodell genannt. Der Versicherer übergibt die Verbraucherinformation bereits vor Vertragsschluß. Er erfüllt damit seine Verpflichtung aus $§ 10$ a VAG. Der Versicherungsvertrag kommt zustande, wenn das Versicherungsunternehmen den Antrag des Versicherungsnehmers annimmt ${ }^{84}$. Im Internet kann das Versicherungsunternehmen diese Vorabinformationen aber nicht erfüllen. Das Antragsmodell kann daher zum Online-Vertragsschluß mit natürlichen Personen nicht genutzt werden. Nur in den Fällen, bei denen eine Verbraucherinformation nach $\S 10$ a VAG nicht notwendig und $\S 5$ a $\mathrm{VVG}^{85}$ daher nicht anwendbar ist, kann ein vollständiger Vertragsschluß im Internet erfolgen ${ }^{86}$, wenn eine Annahme durch das Versicherungsunternehmen erfolgt.

Ein Angebot unter Abwesenden kann gemäß $§ 147$ Abs. 2 BGB nur bis zu dem Zeitpunkt angenommen werden, in welchem der Anbietende eine Antwort unter regelmäßigen Umständen erwarten darf. Der dem Versicherungsunternehmen verbleibende Zeitraum kann daher z.B. einige Tage betragen, wenn das elektronische Angebot noch durch einen Sachbearbeiter zu prüfen ist. Die Annahme kann dann per E-Mail erklärt werden. Der Versicherungsnehmer muß dann noch die Möglichkeit der Kenntnisnahme von der Annahme haben. Handelt es sich bei den Versicherungsnehmern um juristische Personen, so nehmen diese am Geschäftsverkehr teil. Sie müssen daher ihren elektronischen Briefkasten regelmäßig leeren. Für Privatleute haben sich allerdings noch keine festen Regelungen zum Leeren des elektronischen Briefkastens gebildet. Wer allerdings das Internet oder eine E-Mail im

$84 \quad$ Lorenz, ZVersWiss 1995, S. 103 (108).

85 Umstritten ist allerdings, ob $\S 5$ a Abs. 1 VVG immer eine körperlich Übergabe der AVB verlangt oder, ob er diese Verpflichtung nach $\S 10$ a VAG nur betont, vgl. Schirmer, VersR 1996, S. 1045 ( 1049), VerBAV 95, 283 mwN.

86 Gleiches gilt auch, wenn kein Widerspruchsrecht besteht, Dies ist der Fall, wenn es sich um Versicherungsverträge mit Pensionskassen handelt ( $\$ 5$ a Abs. 1 Satz 2 VVG) oder der Versicherer auf besonderen Antrag des Versicherungsnehmers sofortigen Versicherungsschutz gewährt ( $\$ 5$ a Abs. 3 VVG). Die Vertragspartner müssen aber im letzten Fall einen Verzicht auf die Überlassung der AVB und der Verbraucherinformation vereinbaren. 
Rechtsverkehr einsetzt, muß auch in regelmäßigen Zeitabständen nachschauen, ob bei ihm elektronische Willenserklärungen angekommen $\operatorname{sind}^{87}$.

Im Regelfall erfolgt die Annahme auf Seiten des Versicherungsunternehmens durch eine Datenverarbeitungsanlage mittels eines Programmabgleichs. Bei der von der Datenverarbeitungsanlage erzeugten Annahmeerklärung handelt es sich aber nicht um das Resultat einer menschlichen Willensbildung. Da der Progammablauf aber durch die Programmierung auf einem menschlichen Willen beruht, ist die vom Computer erzeugte Erklärung als menschliche Willenserklärung zu sehen ${ }^{88}$. Die Annahme des Angebots durch den Computer erfolgt daher nur kurze Zeit nach Zugang des Angebot durch eine elektronische Mitteilung auf dem Bildschirm des Versicherungsnehmers. Mit dem Zugang der Annahme ist die letzte Voraussetzung für einen Vertragsabschluß erfüllt.

Es gibt aber auch Fälle, in denen die Erklärung einer Annahme nicht notwendig ist. So besteht im Bereich der Kfz-Haftpflichtversicherung ein Kontrahierungszwang. Gemäß $§ 5$ Abs. 3 Satz 1 PflVG gilt ein Versicherungsvertrag dann als abgeschlossen, wenn der Versicherer nicht innerhalb einer Frist von zwei Wochen den Vertragsschluß ablehnt. Eine Annahme des Angebots kann auch konkludent durch die Übersendung des Versicherungsscheins erfolgen.

Wenn die in $\S 10$ a VAG festgelegten Verbraucherinformationen nicht erfolgen müssen und $\S 5$ a VVG nicht anwendbar ist, kann ein Versicherungsvertrag vollständig online zustande kommen. Für das Massengeschäft im Internet kann das Antragsmodell aber nicht genutzt werden.

\section{bb) Policenmodell}

Das mit $§ 5$ a VVG eingeführte neue Vertragsabschlußmodell wird Policenmodell genannt. Es erfaßt die Fälle, in denen der Versicherer den Versicherungsnehmer nicht schon vor Antragstellung gemäß $§ 10$ a VAG informiert hat. Der Vertrag gilt dann auf der Grundlage des

Ernst, NJW-CoR 1997, S. 165 (166); weiterführend zu Zugang von elektronischen Willenserklärungen bei Privatleuten, Gimmy, Vertragsschluß im Internet nach deutschem Recht, in: Gimmy/Kröger aaO Fn. 27.

88 Paefgen, JuS 1988, S. 592 (593); Heun, CR 1994, S. 595 (596); Mehrings, MMR 1998, S. 30 (31) jeweils mwN. 
Versicherungsscheins, der Versicherungsbedingungen und der weiteren für den Vertragsinhalt maßgeblichen Verbraucherinformation als abgeschlossen, wenn der Versicherungsnehmer nicht innerhalb von vierzehn Tagen nach Überlassung der Unterlagen schriftlich widerspricht. Gemäß $§ 5$ a Abs. 2 VVG beginnt die Widerspruchsfrist, wenn der Versicherungsnehmer die Unterlagen vollständig erhalten hat und bei Aushändigung des Versicherungsscheins schriftlich, in drucktechnisch deutlicher Form über das Widerspruchsrecht informiert sowie über den Beginn und die Dauer der Frist belehrt worden ist. Aus Verbraucherschutzgründen kann hier keine Ausnahme vom Schriftlichkeitserfordernis gemacht werden. Im Internet kann daher diese Belehrung nicht erfolgen. Gemäß $§ 5$ a Abs. 2 Satz 4 VVG erlischt das Widerspruchsrecht aber ein Jahr nach Zahlung der ersten Prämie.

Mit dem Policenmodell ist eine Reihe von schwierigen Fragen verbunden, die an dieser Stelle nicht behandelt werden können ${ }^{89}$. Für den Online-Vertragsabschluß muß allerdings geklärt werden, welche rechtlichen Wirkungen das Bestehen des Widerspruchsrechts auf das Zustandekommen des Versicherungsvertrages hat.

Teilweise wird davon ausgegangen, daß $\S 55$ a VVG nur die Einbeziehung der Verbraucherinformationen in den Versicherungsvertrag betreffe, die erst mit Ablauf der Widerspruchsfrist erfolge. Der Vertrag komme aber mit Übersendung der Police als Annahme des Versicherungsunternehmens zustande ${ }^{90}$. Die herrschende Meinung geht davon aus, daß der Vertrag schwebend unwirksam ist, solange die Widerspruchsfrist läuft. Mit Ablauf der Widerspruchsfrist wird der Vertrag auf Grundlage der Police, der AVB und der weiteren Verbraucherinformation wirksam ${ }^{91}$. Für diese Ansicht spricht der Wortlaut des Gesetzestextes und ihr ist daher zu folgen.

Weiterführend: Schimikowski, r+s 1996, S. 1 (3 ff); Wandt, Verbraucherinformation und Vertragsabschluß nach neuem Recht, 1995; Lorenz, ZVersWiss 1995, S. 103 (112 ff.).

90 Hofmann, Die neue Kfz-Versicherung, 1994, S. 28 ff.

91 Schimikowski, r+s 1996, S. 1 (4); Lorenz, ZVersWiss 1995, S. 103 (115); Weyers (Fn 59), Rn. 170; Wandt (Fn. 89), S. 21; Präve, ZfV 1994, S. 374 (382) Innerhalb dieser Meinung ist wiederum umstritten, ob der Vertrag rückwirkend auf den Zeitpunkt des Zugangs der Police wirksam wird oder erst mit Ablauf des Widerspruchsrechts. 
Als Ergebnis für der Abschluß von Versicherungsverträgen im Internet ist daher festzuhalten, daß ein vollständiger Vertragsschluß im virtuellen Raum nicht möglich ist. Es bedarf daher immer noch herkömmlicher Kommunikationsmittel, um die Anforderungen des $\S 10$ a Abs. 2 VAG zu erfüllen. Bis zum Ablauf der Widerspruchsfrist ist ein online geschlossener Versicherungsvertrag schwebend unwirksam.

Die Ausführungen haben gezeigt, daß der Antrag des Versicherungsnehmers auf unterschiedliche Art angenommen werden kann. Es ist daher sinnvoll, den Versicherungsnehmer über die Art und Weise der Annahme auf der Web-Site zu informieren.

\section{e) Das Widerrufs- und Rücktrittsrecht gemäß $\$ 8$ Abs. 4 und 5 VVG}

Für die in $\S 8$ Abs. 4 und 5 VVG festgelegten Widerrufs- und Rücktrittsrechte ist zwischen dem Antrags- und dem Policenmodell zu unterscheiden. Bei dem Policenmodell wird der Versicherungsvertrag gemäß $§ 5$ a VVG geschlossen. Daher steht dem Versicherungsnehmer keines der in $\S 8$ Abs. 4 und 5 aufgeführten Rechte zu (vgl. $\S 8$ Abs. 6 VVG). Da der Versicherungsnehmer ein Widerspruchsrecht gemäß $§ 5$ a VVG hat, ist dieses Ergebnis im Sinne des Verbraucherschutzes auch gerechtfertigt.

Für das Antragsmodell hingegen, das aber nur eingeschränkt im Internet eingesetzt werden kann, gelten die angeführten Rechte. Gemäß $§ 8$ Abs. 4 VVG kann der Versicherungsnehmer seinen Antrag auf Vertragsabschluß bei Verträgen mit einer Laufzeit von über einem Jahr noch 14 Tage nach Unterzeichnung schriftlich widerrufen. Es handelt sich bei dieser Vorschrift um ein Gestaltungsrecht des Versicherungsnehmers, mit dem er einen bereits geschlossenen Vertrag ex tunc vernichten kann. Dieses Widerrufsrecht gilt nach dem Wortlaut der Vorschrift nicht für Lebensversicherungsverträge. Hier normiert $\S 8$ Abs. 5 VVG ein 14-tägiges formloses Rücktrittsrecht für den Versicherungsnehmer. Der Fristablauf beginnt in beiden Fällen mit der vom Versicherungsnehmer $\mathrm{zu}$ unterzeichnenden Belehrung über das Widerrufs- oder Rücktrittsrecht. Ohne Belehrung erlöschen die Rechte einen Monat nach Zahlung der ersten Prämie (vgl. § 8 Abs. 4 Satz 4 und Abs. 5 Satz 4 VVG). Wenn aber der Versicherungsnehmer die Belehrung über seine Rechte mit der Namensunterschrift gemäß $§ 126$ Abs. 1 BGB unterzeichnen muß, so ist dies gegenwärtig im Internet nicht möglich ${ }^{92}$.

92 Vgl. dazu aber die zu erwartenden Änderungen durch den deutschen und europäischen Gesetzgeber, dazu V. 3 und VI. 
Das Schriftlichkeitserfordernis des Widerrufs besagt jedoch nicht, daß das Schriftformerfordernis des $\S 126$ BGB $^{93}$ einzuhalten ist ${ }^{94}$. Hinsichtlich des Widerrufsrechts gemäß § 1 HWiG wird vertreten, daß - abweichend vom Wortlaut - der Widerruf auch mündlich oder gar konkludent erfolgen könne ${ }^{95}$. Das soll auch für das Widerrufsrecht nach $\S 8$ VVG gelten ${ }^{96}$. Damit wäre auch ein Widerruf per E-Mail, bzw. mittels eines auf der Unternehmens-Homepage vorgehaltenen, vom Antragsteller auszufüllenden Formulars möglich.

\section{f) Anfechtung von Willenserklärungen}

Bei der Anfechtung von Willenserklärungen im Internet ist zwischen den menschlichen und den Computerklärungen zu unterscheiden. Bei Eingabe- und Bedienungsfehlern liegt ein Erklärungsirrtum vor. Der Erklärende will eine Erklärung eines solchen Inhalts überhaupt nicht abgeben. Eine Anfechtung nach $\S 119$ Abs. 1, 2. Alt. BGB ist daher möglich ${ }^{97}$.

Bei der Annahme des Vertragsangebots durch ein automatisiertes Verfahren auf Seiten des Versicherungsunternehmens könnten Daten-, Hardware- und Softwarefehler zur Anfechtung der Computererklärung berechtigen. Solche Fehler beeinträchtigen aber nicht die vom Computer erzeugte Willenserklärung. Sie liegen vielmehr im Vorfeld der eigentlichen Willenserklärung und stellen daher einen lediglich unbeachtlichen Motiv- oder Kalkulationsirrtum dar ${ }^{98}$.

Neben Fehlern im Vorfeld von Willenserklärungen und bei deren Abgabe können aber auch Störungen im Internet die Frage nach der Anfechtbarkeit stellen. Das Internet transportiert dabei die Willenserklärungen zwischen den Parteien. Ein Störfall im Server eines OnlineDienstes kann zur Veränderung der Daten führen. In diesen Fällen kann der jeweils Erklärende

\footnotetext{
93 Dazu sogleich supra, S. $31 \mathrm{f}$.

$94 \quad$ Schwenzer, JA 1989, S. 505 (506); Teske, NJW 1991, S. 2793 (2796).

95 Wassermann, Jus 1990, S. 723 (725); Teske ZIP 1986, S. 624 (631); Palandt/Putzo (Fn 56), § 2 HWiG, Rn. 2.

96 Teske, NJW 1991, S. 2793 (2796).

97 Heun, CR 1994, S. 595 (596); Mehrings, MMR 1998, S. 30 (31); Palandt/Heinrichs (Fn 56), § 119 Rn. 10 .

Hoeren, (Fn. 27), Rn. 294; Mehrings, MMR 1998, S. 30 (32); Fritzsche/Malzer, DNotZ 1995 S. 3 (6 f.); Heun, CR 1994, S. 595 (596)
} 
gemäß $§ 120$ BGB anfechten. Die Online-Dienste sind daher als Übermittlungsanstalten im Sinne der Vorschrift zu erfassen ${ }^{99}$.

Für den Fall, daß der Versicherungsnehmer unrichtige Angaben über das versicherte Risiko gemacht hat, wird das Anfechtungsrecht des Versicherungsunternehmens durch die besonderen Regelungen der $\S \S 16-22$ VVG modifiziert.

\section{g) Haustürwiderrufsgesetz (HaustürWG)}

Der Verbraucherschutz bei Vertragsabschluß im Internet könnte durch die Anwendung der Vorschriften des HaustürWG verbessert werden. Das HaustürWG räumt dem Kunden ein Widerrufsrecht ein, wenn seine Willenserklärung auf einer Überraschung oder Überrumpelung an einem in $\S 1$ Abs. 1 HaustürWG genannten Ort beruht. Bei dem Abschluß von Verträgen im Internet beruht die Willenserklärung aber nicht auf einer Überraschung oder Überrumpelung. Der Kunde sucht die Homepage des Unternehmens freiwillig und gezielt auf. Gelegentlich wird eine Anwendung dieses Gesetzes auf Fälle befürwortet, bei denen der Kunde beim Zugriff auf eine Homepage überraschenderweise Werbung vorfindet ${ }^{100}$. Die Diskussion ist für die hier in Frage stehenden Versicherungsverträge aber nicht weiter zu führen. $§ 6$ Nr. 2 HaustürWG stellt die Unanwendbarkeit des HaustürWG auf Versicherungsverträge klar.

\section{Sicherung von Identität des Vertragspartners und Authentizität des Vertragsinhalts}

Neben der technischen und rechtlichen Möglichkeit der Vertragsabwicklung ist Grundvoraussetzung des elektronischen Handels insbesondere die Sicherung der Identität der Vertragspartner sowie der Authentizität des Vertragsinhalts. Der Verkäufer trägt im Internet das Risiko, die Identität des Vertragspartners nicht nachweisen zu können. Im Zweifel wird sich der Verbraucher auf einen Mißbrauch seines Namens durch einen Hacker oder eine beliebige dritte Person berufen. Dies hätte die Folge, daß Ansprüche des Verkäufers gegen ihn ins Leere laufen. Die Nutzung des Internet als Vertriebskanal für Versicherungsdienstleistungen, sowie generell die Etablierung des Electronic Commerce wird also im wesentlichen von der Gewährleistung der Authentizität und der Integrität MMR 1998, S. 30 (32). 
elektronischer Kommunikation, insbesondere elektronischer Dokumente abhängen. Zu diesem Zweck können insbesondere digitale Signaturen eingesetzt werden. Die rechtliche Anerkennung von und das Vertrauen in digitale Signaturen läßt sich nur durch die Inpflichtnahme von Gewährsträgern erreichen, weshalb im Bereich der digitalen Signaturen ein spezifischer rechtlicher Rahmen notwendig ist, der unlängst durch das Signaturgesetz (SigG) und die dazugehörige Signaturverordnung $(\mathrm{SigV})$ geschaffen wurde ${ }^{101}$.

Ziel des Signaturgesetzes ist es, Rahmenbedingungen für die digitale Signaturen zu schaffen, unter denen diese als sicher gelten und Fälschungen zuverlässig festgestellt werden können ( $\$ 1$ Abs. 1 SigG). Eine digitale Signatur ist eine mit einem geheimen Schlüssel zu einem bestimmten elektronischen Dokument erzeugte kryptographische Prüfsumme, die mit einem zweiten, öffentlichen Schlüssel des Urhebers überprüft werden kann ${ }^{102}$. Zu diesem Zweck vergeben die lizenzpflichtigen ${ }^{103}$ Zertifizierungsstellen $^{104}$ auf Antrag Zertifikate, die die Zuordnung eines öffentlichen, jedermann bekannten Signaturschlüssels zu einer natürlichen Person bescheinigen. Das Signaturschlüssel-Zertifikat ist von der Zertifizierungsstelle jederzeit für jeden über öffentlich erreichbare Telekommunikationsverbindungen nachprüfbar und abrufbar zu halten ( $\$ 4$ Abs. 5 S. 3 SigG). Dieses Zertifikat enthält unter anderem den Namen des Schlüssel-Inhabers und den ihm zugeordneten öffentlichen Signaturschlüssel, so daß jederzeit eine Überprüfung der Identität des Absenders eines elektronischen Dokumentes sowie die Integrität des Dokumentes selbst überprüft werden kann. Es ist Unternehmen beim Vertragsabschluß über das Internet deshalb anzuraten, von den Vertragspartnern die Verwendung digitaler Signaturen zu verlangen.

\section{Gerichtliche Durchsetzbarkeit / Schriftformerfordernis}

Damit ist aber noch nichts über den Beweiswert der elektronisch übermittelten

101 Vgl. dazu: Roßnagel, MMR 1998, S. 75; ders., DuD 1997, S. 75.

102 Zur technischen Funktionsweise im einzelnen vgl.: Pordesch/Nissen, CR 1995, S. 562.

103 Die Errichtung der Genehmigungsbehörde bei der Regulierungsbehörde für Telekommunikation und Post steht unmittelbar bevor. 
Willenserklärungen gesagt. Die Schwierigkeiten beginnen, wenn es an die gerichtliche Durchsetzbarkeit der vertraglich gesicherten Ansprüche geht. Nach vorherrschender Auffassung können die digital erstellten Vertragsunterlagen - trotz digitaler Signatur - nicht wie ihr analoges Pendant als Privaturkunde im Sinne von $\S 416$ ZPO anerkannt werden ${ }^{105}$, mit der Folge, daß der Online-Vertrag keinen Strengbeweis darstellt, dessen Beweisregeln das Gericht binden (vgl. § 286 Abs. 2; §§ 415 - 418; § 440 Abs. 2 ZPO). Der Grund dafür ist die fehlende Verkörperung der Erklärung sowie das Fehlen der Unterschrift. Der Online-Vertrag unterliegt lediglich der freien Beweiswürdigung des Gerichts (vgl. § 286 Abs. 1 ZPO). Frühere Bestrebungen, im Rahmen des IuKDG auch die ZPO insoweit zu ändern, als die Urkundseigenschaft eines mit einer digitalen Signatur versehenen, digitalen Dokumentes anerkannt wird, konnten nicht verwirklicht werden. Ein online geschlossener und digital signierter Vertrag leidet somit an einem erheblichen Mangel gegenüber herkömmlich geschlossenen Verträgen.

Dennoch sollte man sich davor hüten, sogleich das Kind mit dem Bade auszuschütten. Es besteht die berechtigte Hoffnung, daß die Gerichte den digitalen Signaturen in der Praxis einen hohen Beweiswert zuerkennen werden. Zum einen, weil die zum Einsatz kommenden kryptographischen Verfahren ein hohes Maß an Sicherheit gewähren, zum anderen, weil zu erwarten ist, daß mit den unabhängigen Zertifizierungsstellen eine hinreichend verläßliche Sicherungsinfrastruktur geschaffen wird, die geeignet ist, Vertrauen in die Sicherheit digitaler Signaturen zu schaffen ${ }^{106}$.

Überdies steht eine Änderung des geltenden Rechts ins Haus, die digitale Signaturen in rechtlicher Hinsicht erheblich aufwerten wird. Schon Anfang 1997 hat das

\footnotetext{
105 Roßnagel, MMR 1998, S. 331 (337); Hoeren, in: Schriftenreihe des Instituts für Rundfunkrecht an der Universität zu Köln, Band 71, S. 57 (71); Ebbing, CR 1996, S. 271 (277); Heun, CR 1995. S. 2 (3). für eine Richtlinie des Europäischen Parlaments und des Rates über gemeinsame Rahmenbedingungen für elektronische Signaturen, KOM (1998) 297 endg., in dessen Anhang II allgemeine Anforderungen an elektronische Signaturen kodifiziert sind. Signaturprodukte, für die die Kommission Referenznummern gem. Art. 3 Abs. 3 RL-E vergeben hat, gelten als vertrauenswürdig im Sinne dieses Anhangs. Gemäß Art. 9 RL-E kann die Kommission in Zusammenarbeit mit dem Ausschuß für elektronische Signaturen die allgemeinen Sicherheitsanforderungen fortschreiben. Optimistisch auch
} Ebbing, CR 1996, S. 271 (274, 278). 
Bundesjustizministerium in einem Entwurf eines Änderungsgesetzes zum BGB die Einfügung eines § 126 a BGB vorgeschlagen, der das Schriftformerfordernis des § 126 BGB durch ein „Textformerfordernis“ ergänzt. Die gesetzliche Schriftform erfordert eine eigenhändige Namensunterschrift des Ausstellers. Eine Textform im Sinne der geplanten Vorschrift läge bereits dann vor, wenn der Text in Schriftzeichen lesbar und der Erklärende erkennbar ist. Die mittels Telekommunikation übermittelten Dokumente erfüllen diese Textform, wenn sie durch Umwandlung in Schriftzeichen lesbar gemacht werden können. Die Erkennbarkeit des Ausstellers könnte durch eine digitale Signatur gewährleistet werden.

Ein paralleler Vorstoß besteht auf europäischer Ebene. Gemäß Art. 5 Abs. 2 des

Richtlinienentwurfs über gemeinsame Rahmenbedingungen für elektronische Signaturen ${ }^{107}$ sollen elektronische Signaturen, die auf einem qualifizierten Zertifikat beruhen, „das rechtliche Erfordernis einer handschriftlichen Unterschrift erfüllen“.

Mit Inkrafttreten dieser Gesetzesänderung ${ }^{108}$ würde die letzte bedeutende Beschränkung des Electronic Commerce beseitigt. Mit der Gleichstellung von digitaler Signatur und handschriftlicher Unterschrift würde das wesentliche Kriterium für die Urkundseigenschaft im Sinne von $§ 416$ ZPO erfüllt, so daß der Online-Vertrag auch prozeßrechtlich nicht länger als „Vertrag zweiter Klasse“ anzusehen wäre.

Insgesamt ist somit nach geltendem Recht ein vollständig virtualisierter Abschluß eines Versicherungsvertrages noch nicht möglich. Neben den bereits skizzierten Rechtsentwicklungen besteht weitere Aussicht auf eine Anpassung der Gesetzeslage durch die europäische Rechtssetzung zu Vertragsabschlüssen im Fernabsatz.

\section{Anwendbarkeit der Fernabsatzrichtlinie auf Versicherungsverträge}

Das Europäische Parlament und der Rat haben am 20.5.1997 eine Richtlinie über den Verbraucherschutz bei Vertragsabschlüssen im Fernabsatz erlassen ${ }^{109}$. Die Richtlinie richtet 
sich an die Mitgliedstaaten und bedarf zu ihrer Geltung noch einer Umsetzung durch die nationalen Gesetzgebungsinstanzen. Diese muß gemäß Art. 15 Abs. 1 der Richtlinie bis zum 5.6.2000 erfolgt sein.

\section{Inhaltsüberblick}

Zum Fernabsatz zählen neben den modernen Distanzvertriebsformen wie TV-Shopping, Internet und E-Mail-Vertrieb auch der klassische Versandhandel ${ }^{110}$. Es werden daher alle Vertriebsformen erfaßt, bei denen ein Vertragsschluß erfolgt, ohne daß eine der Parteien physisch anwesend wäre. Die Verbraucher sollen durch einen EU-weiten Mindeststandard vor einer Übervorteilung und einer erschwerten Durchsetzung ihrer Ansprüche bei grenzüberschreitendem Waren- und Dienstleistungsverkehr geschützt werden ${ }^{111}$. Der Verbraucherschutz wird durch vorgeschriebene Verbraucherinformationen und ein Widerrufsrecht gewährleistet.

Art. 4 der Richtlinie sieht umfangreiche Informationspflichten vor dem Vertragsabschluß vor. Der Verbraucher ist u.a. über die Identität des Lieferers, seiner Anschrift, wesentliche Produkteigenschaften, den Preis und die Lieferkosten aufzuklären. Der Lieferer muß gemäß Art. 5 Abs. 1 seinen Informationspflichten gegenüber dem Verbraucher nachkommen, indem er die in Art. 4 genannten Angaben dem Verbraucher schriftlich oder auf Datenträger verfügbar macht.

Art. 6 Abs. 1 der Richtlinie billigt dem Verbraucher innerhalb von sieben Werktagen ${ }^{112}$ ein Widerrufsrecht ohne Angaben von Gründen zu. Der Fristbeginn bestimmt sich nach der Erfüllung der in Art. 5 genannten Informationspflichten durch den Lieferer. Hat der Lieferer die Informationspflicht nach Art. 5 nicht erfüllt, so kann der Verbraucher den Vertragsabschluß innerhalb einer Frist von drei Monaten ohne Angaben von Gründen widerrufen. Die Frist

Anhang I zur Richtlinie, ABl. EG Nr. L 144 vom 4.6.1997, S. 27.

Zum Inhalt dieser Richtlinie weiterführend Martinek, NJW 1998, S. 207 f; Reich, EuZW 1997, S. 581 ff; Bermanseder, MMR 1998, S. 342 ff; Kröger, Electronic Commerce, in: Gimmy/Kröger; aaO Fn. 27. Fristberechnung erhebliche Divergenzen ergeben, Reich, EuZW 1997, S. 581 (585). Hier sollte der europäische Gesetzgeber auf eine einheitliche Fristberechnung hinwirken. 
beginnt bei Waren mit Wareneingang beim Verbraucher oder bei Dienstleitungen mit Vertragsabschluß. Das Widerrufsrecht ist notwendig, da der Verbraucher in der Praxis keine Möglichkeit hat, vor Abschluß des Vertrages das Erzeugnis zu sehen oder die Eigenschaften der Dienstleistungen im einzelnen zur Kenntnis zu nehmen ${ }^{113}$.

\section{Anwendung auf Versicherungsverträge}

Die Fernabsatzrichtlinie gewährleistet einen wirksamen virtuellen Vertragsabschluß und einen EU-weiten Mindeststandard im Verbraucherschutz. Es ist daher bedauerlich, daß gerade Finanzdienstleistungen gemäß Art. 3 Abs. 1 aus dem Anwendungsbereich der Richtlinie herausgenommen wurden. Die Finanzdienstleistungen wurden im Anhang II der Richtlinie ${ }^{114}$ näher aufgeführt. Zu ihnen zählt auch das Versicherungs- und Rückversicherungsgeschäft. Dabei waren Finanzdienstleistungen einschließlich der Versicherungsverträge in den ersten Entwürfen der Fernabsatzrichtlinie enthalten ${ }^{115}$. Unter Hinweis auf die bisher erfolgten Liberalisierungsmaßnahmen im Bereich der Finanzdienstleistungen wurden diese aber 1995 endgültig aus dem Anwendungsbereich der Fernabsatzrichtlinie herausgenommen ${ }^{116}$. Finanzdienstleistungen wurden nach dem Binnenmarktprogramm liberalisiert. Die Finanzdienstleistungsunternehmen unterliegen dem Herkunftslandprinizip, d.h. sie werden von den nationalen Behörden beaufsichtigt. Sie dürfen aber im Wege der Sekundärniederlassung oder im freien Dienstleistungsverkehr ihre Produkte europaweit anbieten. Wenn im Rahmen der Liberalisierung ein bestimmter Verbraucherschutz eingeführt wurde, etwa ein Widerrufsrecht gemäß Art. 30 der 3. Richtlinie Lebensversicherung 92/96/EWG ${ }^{117}$, so beruht dies auf den Besonderheiten des Produkts. Die Gefahren, die denen mit der Fernabsatzrichtlinie gewehrt werden sollen, wurden durch die liberalisierenden Richtlinien aber nicht berücksichtigt $^{118}$. Es ist daher unverständlich, daß Versicherungsverträge und andere

AB1. EG Nr. L 144 vom 4.6.1997, S. 20.

ABl. EG Nr. L 144 vom 4.6.1997, S. 26.

Schirnding, FLF 1998, S. 11 (11). Zur Anwendung der ersten Entwürfe der Fernabsatzrichtlinie auf Versicherungsverträge Präve, VersR 1993, S. 1066.

AB1. EG Nr. C. 288 vom 30.10.1995.

ABl. EG Nr. L. 360 vom 9.12.1992.

Reich, EuZW 1997, S. 581 (583). 
Finanzdienstleistungen aus dem Anwendungsbereich der Fernabsatzrichtlinie ausgenommen wurden $^{119}$.

\section{Geplante Fernvertragsrichtlinie über Finanzdienstleistungen}

Der europäische Gesetzgeber ist sich aber bewußt, daß der Fernvertrieb von Finanzdienstleistungen einer Regelung bedarf ${ }^{120}$. Gegenwärtig liegen daher jeweils eigenständige Entwürfe der Generaldirektionen XV und XXIV über eine Fernvertragsrichtlinie über Finanzdienstleistungen $\operatorname{vor}^{121}$. Da es sich bei den Richtlinienentwürfen um nicht öffentliche Entwürfe handelt und zum gegenwärtigen Zeitpunkt noch nicht ersichtlich ist, wann und mit welchem Inhalt eine Fernvertragsrichtlinie über Finanzdienstleistungen verabschiedet wird, soll nur ein kurzer Inhaltsüberblick gegeben werden.

Die Richtlinienentwürfe übernehmen den Aufbau der Fernabsatzrichtlinie. Die Anbieter von Finanzdienstleistungen, zu denen alle Bank-, Versicherungs-, Anlage-, und PensionsfondsDienstleistungen zählen, treffen umfangreiche vorvertragliche Informationspflichten gegenüber dem Kunden.

Zu den Kunden im Sinne des Richtlinienentwurfs der Generaldirektion XV zählen aber nicht andere Finanzinstitute, internationale Organisationen, staatliche Stellen und Gesellschaften mit mehr als 50 Beschäftigten, einer Bilanzsumme von mindestens 2500000 ECU und einem Nettoumsatzerlös von mindestens 5000000 ECU. Es erscheint aber überaus praxisfern, den Anwendungsbereich der Richtlinie auf diese Art $\mathrm{zu}$ begrenzen. Der Anbieter der Finanzdienstleistungen müßte umfangreiche Daten erheben, um herauszufinden, ob der Kunde in den Anwendungsbereich der Richtlinie fällt oder nicht ${ }^{122}$. Da Verbraucherschutz immer auf den Schutz des privaten Verbrauchers abzielt, reicht es aus, wenn geschäftsmäßiges, gewerbliches oder berufliches Handeln des Kunden aus dem Anwendungsbereich des Richtlinienentwurfs ausgeschlossen wird.

\footnotetext{
119 kritisch auch Hoeren, NJW 1998, S. 2849 (2851 f.).

$120 \quad$ KOM 96 (209) endg., S. 1.

$121 \quad$ Schirnding, FLF 1998. S. 11 (11 ff.).

$122 \quad$ Schirnding, FLF 1998, S. 11 (14).
} 
Die Informationspflichten stimmen im wesentlichen mit denen der Fernabsatzrichtlinie überein. Im Gegensatz zur Fernabsatzrichtlinie ist der Kunde neben dem Bestehen eines Widerrufsrechts aber auch über die Bedingungen für eine Vertragsauflösung einschließlich etwaiger Vertragsstrafen $\mathrm{zu}$ informieren. Diese Klausel ist besonders für langlaufende Versicherungsverträge von großer Bedeutung und erweitert den Verbraucherschutz in den Mitgliedstaaten.

Nach Vertragsabschluß sind dem Kunden in schriftlicher oder in anderer, ihm zugänglicher dauerhafter Form die für den Vertrag geltenden Bedingungen mitzuteilen und dabei die vor Vertragsschluß übermittelten Informationen zu bestätigen. Ferner ist der Kunde über die Bedingungen und Verfahrensweisen für die Ausübung des Rücktrittsrechts und über die Einzelheiten hinsichtlich der Beschwerde und Abhilfeverfahren zu informieren. Die Dauer zur Erfüllung der Informationspflichten schwankt in den einzelnen Entwürfen zwischen ein und drei Monaten ${ }^{123}$.

In der Entwürfen zur Finanzdienstleistungsrichtlinie findet sich gleichfalls ein Rücktrittsrecht, das dem der Fernabsatzrichtlinie entspricht. Es gilt aber nicht für solche Finanzdienstleistungsverträge, die sich auf Finanzinstrumente beziehen, deren Kurs von Schwankungen auf dem Finanzmarkt abhängt, die der Anbieter nicht steuern kann ${ }^{124}$. Für Versicherungsverträge ist dieser Ausschluß des Rücktrittsrechts bedeutungslos.

Die geplante Fernvertragsrichtlinie über Finanzdienstleistungen führt für diese im wesentlichen die Rechtslage ein, die für alle anderen Verträge über Waren und Dienstleistungen im Distanzvertrieb schon heute gilt. Geht man daher davon aus, daß Verträge im Fernabsatz einer verbraucherschützenden Regelung bedürfen, ist es überaus fraglich, warum für 
Finanzdienstleistungen ${ }^{125}$ eine eigene Richtlinie geschaffen werden soll ${ }^{126}$. Die getroffenen Unterscheidungen hätten, soweit sie nötig sind, in der „Mutterrichtlinie“ berücksichtigt werden können. Der europäische Gesetzgeber verstärkt mit dem eigenständigen Ansatz lediglich die Anzahl der Rechtsakte, die einzeln und mühsam von den nationalen Gesetzgebern umzusetzen sind. Im Ergebnis wird mit der beabsichtigten Fernvertragsrichtlinie über Finanzdienstleistungen der schon in der Fernabsatzrichtlinie verankerte Verbraucherschutz auch auf den Vertrieb von Versicherungen im Internet ausgedehnt.

Da es aber bei den ersten Expertenanhörungen zu unterschiedlichen Meinungen über die Notwendigkeit der Richtlinie und deren Inhalt gekommen ist, ist mit einem langwierigen Verfahren bis zu Verabschiedung zu rechnen ${ }^{127}$.

\section{Grenzüberschreitender Vertrieb}

Das Kollisionsrecht im Versicherungsvertragsrecht stellt bei der Nutzung des Internets zu grenzüberschreitenden kommerziellen Zwecken die große Unbekannte dar. Die Lösung der vielfältigen Probleme muß hier der weiteren Entwicklung überlassen werden. Grundsätzlich treffen beim grenzüberschreitenden Versicherungsvertrieb aus deutscher Sicht zwei Kollisionssysteme aufeinander. Es handelt sich dabei um die Regelungen des EGBGB, wobei den Art. 3 ff, 27 bis 36 EGBG besondere Beachtung zu schenken ist ${ }^{128}$. Daneben sind die Art. 7 bis 15 EGVVG zu erwähnen. Die beiden Kollisionssysteme sind voneinander abzugrenzen.

Für einen generellen Verbraucherschutz beim Distanzvertrieb von Finanzdienstleistungen sprechen sich Präve, VersR 1993, S. 1066 (1069f) und Reich, EuZW 1997, S. 581 (583) aus. Schirnding, FLF 1998, S. 11 (12) sieht spezielle Gefahren im Distanzvertrieb nicht, sondern befürwortet einen produktbezogenen Verbraucherschutz.

Dies scheint selbst die Kommission nicht zu wissen, vgl. KOM 96 (209), S. 14, da sie mit der geplanten Richtlinie nach eigener Aussage für den Kunden von Finanzdienstleistungen beim Fernverkauf einen Schutz herstellen will, der dem beim Kauf anderer Waren oder Dienstleistungen entspricht. Geschäftsbericht des Bundesaufsichtsamts für das Versicherungswesen 1997, S. 31.

Weiterführend zu den Vorschriften des EGBGB und die Anwendung im Internet: Hoeren, Rechtsfragen des Internets, Rn. 276 ff; Gimmy, Vertragsabschluß im Internet nach deutschem Recht, in: Gimmy/Kröger; aaO Fn. 27; Bachman, Internet und IPR, in: Lehmann (Hrsg.), Internet- und Multimediarecht, S, 169 (173 ff.); Fricke, VersR 1994, S. 773 ff. Zur Einbeziehung von AGBG in internationale Verträge Koehler, MMR 1998, S. 289 (292) jeweils mwN. 
Das EGBGB regelt seine Anwendbarkeit negativ. Es ist gemäß Art. 37 Nr. 4 EGBGB nicht anwendbar auf Verträge, welche ein Risiko abdecken, das innerhalb des Geltungsbereiches des Vertrages zur Gründung der Europäischen Wirtschaftsgemeinschaft oder des Abkommens über den Europäischen Wirtschaftsraum belegen ist. Gemäß $\S 37$ Nr. 4 Satz 2 EGBGB ist die Risikobelegenheit nach dem Recht des Staates, dessen Richter um eine Entscheidung angegangen wird, zu beurteilen. Die Regelungen des EGBGB mit dem Grundsatz der freien Rechtswahl in Art 27 EGBGB und der Schutzvorschrift des $\$ 29$ EGBGB für Verbraucherverträge gelten für Versicherungsverträge, die ein Risiko bedecken, das außerhalb des EWR oder EU belegen ist. Art. 29 Abs. 1 Nr. 1 EGBGB ist hier beim Internet-Vertrieb besondere Bedeutung zu schenken. Die Vorschrift erfaßt solche Verträge, denen vor Vertragsschluß ein ausdrückliches Angebot oder Werbung in diesem Staat vorausgegangen ist und bei denen der Verbraucher in diesem Staat die zum Vertragsschluß erforderliche Handlung vorgenommen hat. Gemäß Art. 29 Abs. 2 EGGBG gilt dann das Recht des Staates, in dem der Verbraucher seinen gewöhnlichen Aufenthalt hat.

In Art. 7 Abs. 1 EGVVG ist - im Gegensatz zu Art 37 Nr. 4 EGBGB - der Anwendungsbereich der Normen positiv mit der Risikobelegenheit in einem Mitgliedsstaat der Europäischen Union und des EWR beschrieben. Gemäß Art. 8 EGVVG ist dann das Recht des Staates anzuwenden, in dem das Risiko belegen ist, wenn der Versicherungsnehmer bei Schließung des Vertrages seinen gewöhnlichen Aufenthalt oder seine Hauptverwaltung in dem Mitgliedstaat hat.

Diese Kurzdarstellung zeigt, daß die Versicherungsunternehmen bei einem grenzüberschreitenden Internet-Vertrieb ggf. eine Vielzahl von Rechtsordnungen beachten müssen $^{129}$. Dies gilt auch für den Abschluß von Versicherungsverträgen innerhalb der EU, da auf eine Harmonisierung des Versicherungsvertragsrechts bisher verzichtet wurde. Für die Unternehmen kann es daher sinnvoll sein, grenzüberschreitende Verträge einzuschränken oder generell abzulehnen.

Noch eklatanter ist das Defizit rechtlicher Steuerung im Internationalen Bereich. Völkerrechtliche verbindliche Regelungen zum electronic commerce fehlen weitestgehend; zaghafte Harmonisierungsbestrebungen bestehen jedoch zuletzt im Rahmen der OECD, vgl. Handelsblatt v. 9.10.98, S. 10; grundlegend: Moos, Die Entwicklung eines supra- und internationalen Rechtsrahmens für das Internet, in: Gimmy/Kröger (Hrsg.), (Fn. 27), Kap.2. 


\section{Versicherungsaufsichtsrecht}

Abschließend soll ein kurzer Überblick über die aufsichtsrechtlichen Probleme des InternetVertriebs von Versicherungsdienstleistungen gegeben werden.

\section{Vertrieb von Versicherungsdienstleistungen im Inland}

Bietet ein Versicherungsunternehmen mit Sitz im Inland im Internet den Abschluß von Versicherungsverträgen an, so bedarf es dazu der Genehmigung durch die Aufsichtsbehörde. Es muß daher eine Erlaubnis zum Geschäftsbetrieb gemäß $§ 5$ Abs. 1 VAG beantragt werden. Dies hat aber nur für eine reine Internet-Versicherung Bedeutung, da alle anderen Versicherungsunternehmen, die auch einen herkömmlichen Vertrieb haben, bereits über eine Genehmigung gemäß $§ 5$ Abs. 1 VAG verfügen.

\section{Europarechtliche Einordnung}

Auch die europarechtliche Einordnung bereitet keine Schwierigkeiten. Der Bereich der Finanzdienstleistungen einschließlich der Versicherungsdienstleistungen wurde bereits durch den europäischen Gesetzgeber liberalisiert. Der Binnenmarkt im Bereich der Finanzdienstleistungen basiert auf den Grundsätzen der Herkunftslandkontrolle und der gegenseitigen Anerkennung, die die Annahme vereinbarter aufsichtsrechtlicher Mindeststandards voraussetzt. Versicherungsunternehmen können daher ihre Dienstleistungen europaweit grenzüberschreitend oder mittels einer Zweigstelle im Zielstaat anbieten. Der Internet-Vertrieb stellt daher für europäische Versicherer die Erbringung einer grenzüberschreitenden Dienstleistung dar. Die erforderliche Zulassung wird für Versicherungen aus dem Bereich der EU oder dem Europäischen Wirtschaftsraum von der zuständigen Behörde des Sitzlandes erteilt ${ }^{130}$.

\section{Rechtliche Einordnung von Unternehmen aus Drittstaaten als Korrespondenzversicherung}

Größere Probleme bestehen beim Online-Auftritt von Versicherungen aus Drittstaaten. Versicherungsunternehmen, die ihren Sitz außerhalb der EU oder des Europäischen 
Wirtschaftsraums haben, sind als sog. Drittlandsunternehmen einzuordnen. Gemäß $\S 105$ Abs. 1 VAG benötigen sie für den Versicherungsvertrieb eine Zulassung, wenn sie im Inland das Direktversicherungsgeschäft durch Mittelspersonen betreiben wollen.

Während bei EU-Versicherern ein gewisser Aufsichtsstandard durch das Prinzip der Herkunftslandkontrolle gesichert ist, stellt sich bei Versicherungen aus Drittstaaten die Frage, wie Verbraucher vor unseriösen Angeboten geschützt werden können. Eine Beaufsichtigung durch eine Aufsichtsbehörde eines EU-Mitgliedstaats ist allerdings nicht effizient möglich, wenn sich die Versicherung in einem Drittstaat befindet. Gegenwärtig hat sich das Bundesaufsichtsamt für das Versicherungswesen noch nicht entschieden, ob es sich bei solchen Online-Auftritten um zulassungsfreie Korrespondenzversicherungen handeln soll. Bei einer Korrespondenzversicherung handelt es sich um eine Versicherung, die durch Korrespondenz zwischen einer Person, die ihren gewöhnlichen Aufenthalt im Inland hat, und einem Versicherungsunternehmen im Ausland zustandekommt, ohne daß dabei ein geschäftsmäßig handelnder Vertreter mitwirkt und dadurch für das ausländische Versicherungsunternehmen Versicherungsgeschäfte betreibt ${ }^{131}$. Eine Tendenz zu einer solchen Einordnung besteht allerdings ${ }^{132}$. Da aber beim Vertrieb von Versicherungen über das Internet auf das Einschalten von Mittelspersonen verzichtet werden kann, liegt der Tatbestand des § 105 Abs. 1 VAG nicht vor.

\section{Verbraucherschutz durch Online-Angebot des BAV}

Wenn auch das BAV oder eine andere europäische Aufsichtsbehörde eine Aufsicht über Versicherungsunternehmen aus Drittstaaten nicht sicher stellen kann, so kann ein gewisser Verbraucherschutz auf einem anderen Wege erfolgen. Das BAV beabsichtigt, auf einer eigenen Homepage die Unternehmen aufzulisten, die der Aufsicht einer deutschen oder europäischen Aufsichtsbehörde unterliegen. Der Verbraucher kann sich dann durch Ansteuern der Homepage des BAV vor Abschluß des Versicherungsvertrages darüber informieren, ob das Versicherungsunternehmen zum Vertrieb von Versicherungen in Deutschland zugelassen ist. Hat er auf diese Weise über den Aufsichtsstandard Kenntnis erlangt, so kann er

\footnotetext{
131 Schmidt, in: Prölss/Schmidt (Fn. 130), § 105, Rn. 10.

132 VerBAV 1998, S. 140; kritisch zur Einordnung als Korrespondenzversicherung beim Btx-Vertrieb Schmidt, in: Prölss/Schmidt (Fn. 130), § 105, Rn. 12.
} 
eigenverantwortlich entscheiden, ob er durch die freiwillige Versicherungsnahme in einem Drittland das Risiko eines unbekannten oder gar nicht vorhandenen Aufsichtssystems eingehen will.

\section{Abschließende Bewertung}

Das Internet kann für Versicherungen eine interessante und kostengünstige Ergänzung der traditionellen Vertriebswege sein und sollte in der strategischen Planung jedes Versicherers eine Rolle spielen. Grundsätzliche rechtliche Bedenken gegen den Internet-Vertrieb von Versicherungsdienstleistungen bestehen nicht. Das Policenmodell behindert aber noch den Versicherungsvertragsabschluß im Internet für das Massengeschäft. Es ist daher bedauerlich, daß die Fernabsatzrichtlinie dieses Problem nicht gelöst hat. Für einen vollständigen Vertragsabschluß im virtuellen Raum bleibt daher zu hoffen, daß die Fernvertragsrichtlinie zu Finanzdienstleistungen möglichst bald verabschiedet und dann zügig vom deutschen Gesetzgeber umgesetzt wird. Außerdem müßte die Infrastruktur zur Verwendung rechtlich anerkannter digitaler Signaturen möglichst bald geschaffen werden. Bei Versicherungsverträgen mit Auslandsberührung bestehen größere Unsicherheiten; die Versicherungsunternehmen müssen jeweils genau prüfen, ob sich der Vertragsabschluß lohnt, oder eine Kontrahierung eher ausgeschlossen werden sollte. 